OPEN ACCESS

Edited by:

Rayne Rouce,

Baylor College of Medicine

United States

Reviewed by:

Hongbin Wang,

California Northstate University,

United States

Chiara Bonini,

Vita-Salute San Raffaele University,

Italy

*Correspondence:

Masoud Soleimani

soleim_m@modares.ac.ir

Jafar Kiani

kiani.ja@iums.ac.ir;

ja.kiani@gmail.com

tThese authors share first

co-authorship

\#ORCID:

Mohammadreza Azangou-Khyavy orcid.org/0000-0002-4543-4633

Mobina Ghasemi

orcid.org/0000-0003-0752-2410

Javad Khanali

orcid.org/0000-0002-9853-454X

Melika Boroomand-Saboor orcid.org/0000-0001-9031-3454

Monire Jamalkhah

orcid.org/0000-0002-0737-6313

Masoud Soleiman

orcid.org/0000-0003-1972-7771

Jafar Kiani

orcid.org/0000-0002-5907-5846

Specialty section:

This article was submitted to

Cancer Immunity and Immunotherapy,

a section of the journal

Frontiers in Immunology

Received: 05 March 2020

Accepted: 29 July 2020

Published: 29 September 2020

Citation:

Azangou-Khyavy M, Ghasemi M, Khanali J, Boroomand-Saboor M,

Jamalkhah $M$, Soleimani $M$ and Kiani J (2020) CRISPR/Cas: From Tumor Gene Editing to T Cell-Based

Immunotherapy of Cancer.

Front. Immunol. 11:2062.

doi: 10.3389/fimmu.2020.02062

\section{CRISPR/Cas: From Tumor Gene Editing to T Cell-Based Immunotherapy of Cancer}

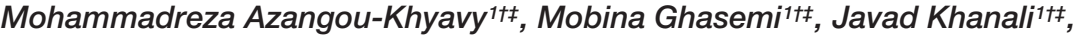 \\ Melika Boroomand-Saboor ${ }^{1 \dagger \neq}$, Monire Jamalkhah ${ }^{2 \dagger \neq}$, Masoud Soleimani ${ }^{* \neq}$ and \\ Jafar Kiani ${ }^{4,5 * \neq}$ \\ ${ }^{1}$ Student Research Committee, Shahid Beheshti University of Medical Sciences, Tehran, Iran, ${ }^{2}$ Department \\ of Biotechnology, College of Science, University of Tehran, Tehran, Iran, ${ }^{3}$ Hematology Department, Faculty of Medical \\ Sciences, Tarbiat Modares University, Tehran, Iran, ${ }^{4}$ Oncopathology Research Center, Iran University of Medical Sciences, \\ Tehran, Iran, ${ }^{5}$ Department of Molecular Medicine, Faculty of Advanced Technologies in Medicine, Iran University of Medical \\ Sciences, Tehran, Iran
}

The clustered regularly interspaced short palindromic repeats system has demonstrated considerable advantages over other nuclease-based genome editing tools due to its high accuracy, efficiency, and strong specificity. Given that cancer is caused by an excessive accumulation of mutations that lead to the activation of oncogenes and inactivation of tumor suppressor genes, the CRISPR/Cas9 system is a therapy of choice for tumor genome editing and treatment. In defining its superior use, we have reviewed the novel applications of the CRISPR genome editing tool in discovering, sorting, and prioritizing targets for subsequent interventions, and passing different hurdles of cancer treatment such as epigenetic alterations and drug resistance. Moreover, we have reviewed the breakthroughs precipitated by the CRISPR system in the field of cancer immunotherapy, such as identification of immune system-tumor interplay, production of universal Chimeric Antigen Receptor T cells, inhibition of immune checkpoint inhibitors, and Oncolytic Virotherapy. The existing challenges and limitations, as well as the prospects of CRISPR based systems, are also discussed.

Keywords: CRISPR, cancer treatment, gene therapy, cancer immunotherapy, CAR T cell therapy, genome-wide screening assays, oncolytic virotherapy

\section{INTRODUCTION}

In recent years, various genetic manipulation techniques have been developed which involve DNA repair mechanisms that incorporate site-specific modifications into a cell's genome. These techniques have made diverse genome alterations in a site-specific manner possible, as they are able to edit tumor cells' genome to induce apoptosis, reduce drug resistance, and restore mutant genes. They also can be employed in immune system genetic manipulation to potentiate antitumor immune responses (1-4). Clustered Regulatory Interspaced Short Palindromic Repeats (CRISPR) is a novel mammalian cells' genome editing technique derived from archaeal and bacterial antiviral defense systems. Due to its exceptional potential and efficacy, this platform has defined a new era, tipping the balance of cancer treatment in favor of genetic manipulation of the tumor and immune cells. CRISPR is preferentially exploited to interrogate various genes and signaling pathways' functions, leading to the discovery of new therapeutic targets. In this review, we will 
discuss the CRISPR technique and explore its recent applications in discovering new targets in cancer therapy and overcoming different hurdles of cancer treatment, such as oncogenes, epigenetic dysregulations, and drug resistance. We will also dissect its redemptive potential in immunotherapy. The subtitles that will be discussed later in this review are shown in Figure 1.

\section{GENOME EDITING TECHNIQUES: ZFNs, TALENS, AND CRISPR}

Up until now, three nuclease-based systems have been developed with ubiquitous genome editing applications and with the capacity to be engineered for specific sequence targeting. Zinc Finger Nucleases (ZFNs) are constructed by fusing zinc finger protein motifs with the DNA cleavage domain of FOK1 endonuclease. Zinc fingers are small protein motifs that can bind in the major groove of DNA in a sequence-specific manner. Multiple zinc finger modules can be assembled into a more massive complex to achieve higher specificity. As FOK1 needs homodimerization at the target site to cleave DNA, two separate zinc finger modules possessing adjacent target sites (Figure 2A) are incorporated. Generally, ZFNs possesses the potential to target sequences with 9-18 bp length (5-7).

Transcription Activator-Like Effector Nuclease (TALEN) is another nuclease-based system similar to ZFN in many aspects. TALENs are constructed by the chimeric fusion of the FOK1 cleavage domain to the complex of TALE DNA-binding modules. Each module is comprised of 34-35 amino acids with the ability to recognize a single base pair on the plus side. These TALE proteins, which were first discovered in Xanthomonas bacteria, can be designed to target 7-34 bp-long DNA targets (Figure 2A) (8-10).

The third system, CRISPR/Cas, was first introduced by Cong et al. and Mali et al. as a mammalian cell genome editing platform (11-13). This discovery has led to dramatic improvements in genetic manipulation specificity and efficacy. Moreover, it has expanded the application spectrum beyond mere genome editing, and new prospects of therapeutic and research purposes are being explored (14-17).

CRISPR/Cas constitute adoptive immunity against bacteriophages, transposable elements, and plasmids in Bacteria and Archaea. Upon pathogen invasion, these organisms insert segments of the invader genetic material into the CRISPR loci as new spacers within two repeat sequences. In cases of future invasions, these loci are transcribed into pre-crRNA and subsequently processed by Cas and other cellular agents into its mature form, CRISPR RNA (crRNA) (18-20). After that, the (crRNA)/Cas protein(s) complex detects mobile genetic elements with sequence specificity primarily arising from Watson-Crick base pairing between crRNA and target DNA (21).

There are two major classes and several types of CRISPR systems based on differences in components and mechanisms of action. In contrast to other classes, which rely on many effector proteins for RNA-guided target cleavage, the class 2 system relies on only one RNA guided endonuclease (Cas9 in type 2 and Cpf1 in type5), turning it into a more straight-forward genetic-altering device. Thus, researchers have widely used this class of CRISPR system $(14,19-21)$.

CRISPR/Cas9 is the most extensively used CRISPR system in genome editing techniques. This system employs Cas9 as its RNA-guided endonuclease and crRNA as a guiding RNA, as well as trans-activating CRISPR RNA (tracrRNA). tracrRNA is a noncoding RNA and is critical for crRNA processing, Cas9 binding, and target DNA breaking (Figures 2B,C) (in the type5 CRISPR system, Cpf1 can detect and cleave the target DNA independently of tracrRNA) (22-24).

Catalytically inactivated Cas9, dCas9, has many applications beyond genome editing. As dCas9 cannot break DNA strands, it is used as a sequence-specific DNA binding element. Through binding different effector parts to dCas9, it can be used as a transcription inhibitor or activator or even epigenetic modulator (16). These applications and some other applications are summarized in Figure 3.

\section{CRISPR Versus ZFN and TALEN}

CRISPR has demonstrated considerable advantages over ZFNs and TALENs as it functions through DNA-RNA interaction. Since ZFN and TALEN rely on protein-DNA interaction for their sequence specificity, targeting a new site requires engineering a new protein, hence constraining these tools' implementation in high-throughput applications. Additionally, the high molecular weights of these proteins in correspondence to the length of the sequence they target hinders multi-targeting during a single process of genetic engineering, which is mainly due to the genetic delivery limitations to the host and the metabolic burden they impose on the target cell (25). In contrast, the CRISPR system can be retargeted to a new site only by changing the guide RNA sequence $(4,21)$. Moreover, multiple site-specific genetic alterations are possible through the delivery of a single form of Cas and multiple sgRNAs requiring fewer macromolecules than multiple ZFNs and TALENs, which in turn results in lower cellular toxicity. Besides, some have declared that CRISPR possesses higher genome editing efficiency relative to ZFNs and TALENs $(11,26,27)$.

\section{CRISPR/Cas AND TUMOR CELL MANIPULATION}

Characterizing and targeting genes responsible for tumorigenesis and cancer progression remains crucial yet challenging. Alterations in regulations of these genes, dubbed as oncogenes and tumor suppressors, mediate resistance to therapy and cancer progression. The CRISPR/Cas system has been exploited for discovering and introducing such genes and their cognate pathways as novel targets and has served as a powerful tool for cancer gene therapy.

\section{Gene Therapy via CRISPR/Cas}

The excessive accumulation of specific mutations leads to biological hallmarks of a malignant phenotype (28). Through gene therapy, CRISPR/Cas9 technology is applicable in 


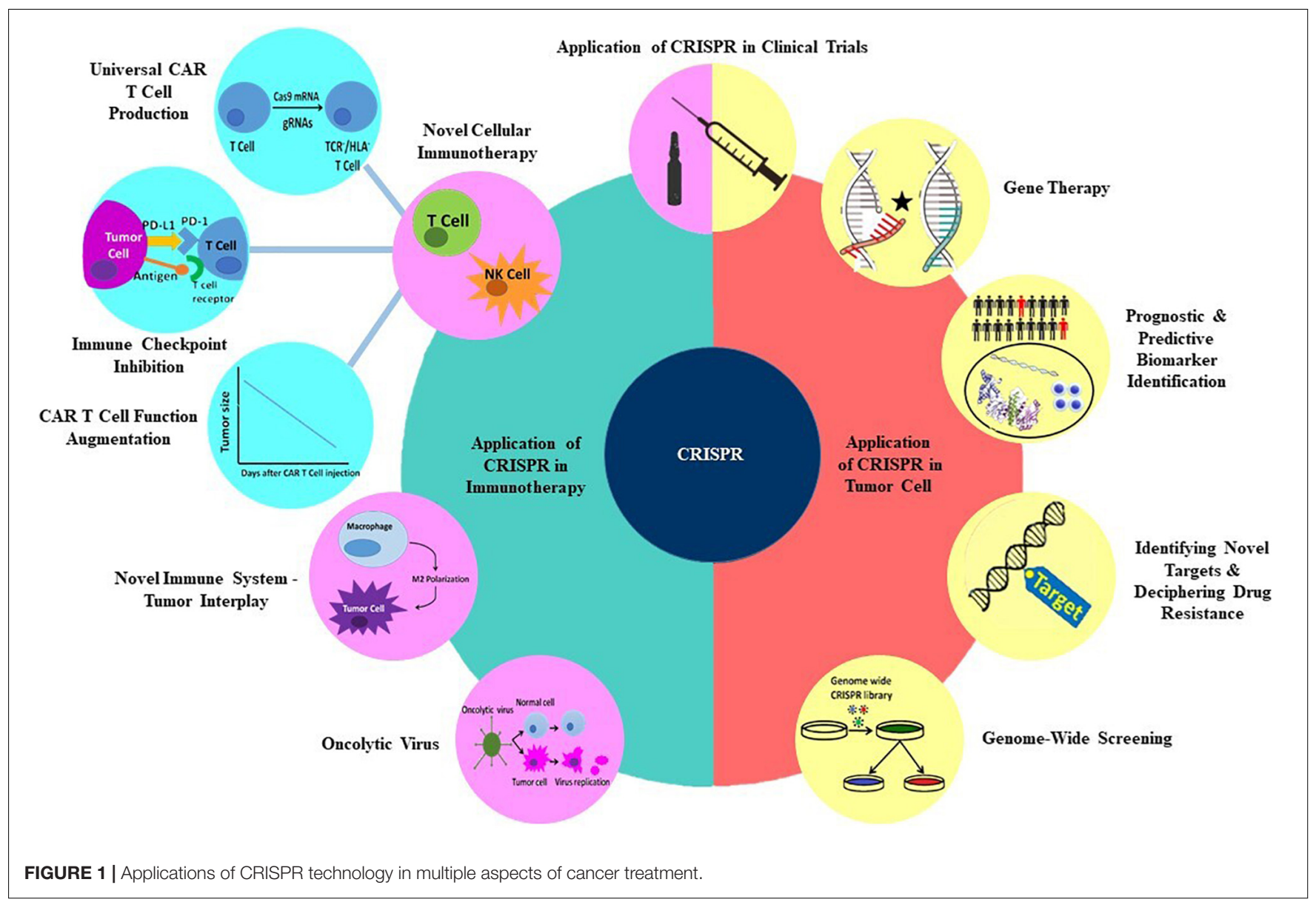

oncogenes' inactivation or restoration of tumor suppressors and apoptotic and immune-stimulatory functions.

A common characteristic of many tumor cells is a mutation in tumor protein 53 (TP53 in human or TrP53 in mice). This gene encodes the tumor suppressor protein p53, which is responsible for halting cell proliferation in response to internal stress and abnormality inputs (29). Almost half of human malignancies are harboring an altered form of TP53 (2). Albers et al. showed that CRISPR/Cas9-mediated inactivation of Transformation Related Protein 53 (TrP53) and expression of oncogene H-Ras led to cellular transformation and tumor formation in a xenograft model (30).

Restoring the mutated TP53 to its wild type function using various compounds can induce apoptosis and senescence in tumor cells. Chira et al. envisioned a novel Tp53 therapeutic concept, capable of replacing the entire mutant locus of TP53 ( $\sim 20.5 \mathrm{~kb}$ in length) with its functional cDNA version through homologous recombination. This recombination required the expression of two sgRNAs (single guide RNA comprising crRNA and tracrRNA fusion) binding to upstream and downstream flanking sites of the TP53 mutant locus. They designed a hybrid of an Adeno-Associated Virus and a bacterioPhage (AAVP) directed to tumor cells. Hence, the design increased the specificity, and it could also possess an inducible functionally through the administration of a simple antibiotic like doxycycline. The intravenous administration of this therapeutic vector yielded limited side effects and increased distribution, leading to sustained expression of p53 and tumor regression even in distant metastatic tumor sites (2).

Human Estrogen Receptor 2 (HER2) gene is a well-known oncogene and is over-expressed in some cancers, such as breast cancer, serving as a therapeutic target for Herceptin (Trastuzumab). As an alternative, Wang et al. utilized a novel strategy to target the oncogene HER2 with the CRISPR/Cas9 system. Co-expression of Cas9 and three sgRNAs targeting HER2 exons 5, 10, and 12 significantly reduced cell growth and tumorigenicity in Her2-positive breast cancer cells (31). One advantage of employing CRISPR/Cas9-mediated HER2 downregulation over conventional therapeutics such as monoclonal antibodies (mAbs) is the simplicity of designing new guide RNAs for targeting new mutations in the case of resistance. The development of conventional therapeutics would, on the other hand, require a new drug discovery program, which is a time-consuming and laborious practice.

Epidermal Growth Factor Receptor (EGFR) is a glycoprotein anchored to the cell's membrane and has an intracellular tyrosine kinase domain. Constitutive tyrosine kinase activation due to genetic mutation causes cancer formation and progression. Although Tyrosine Kinase Inhibitors (TKIs) have been the therapeutic choice for EGFR-expressing malignancies, resistance 


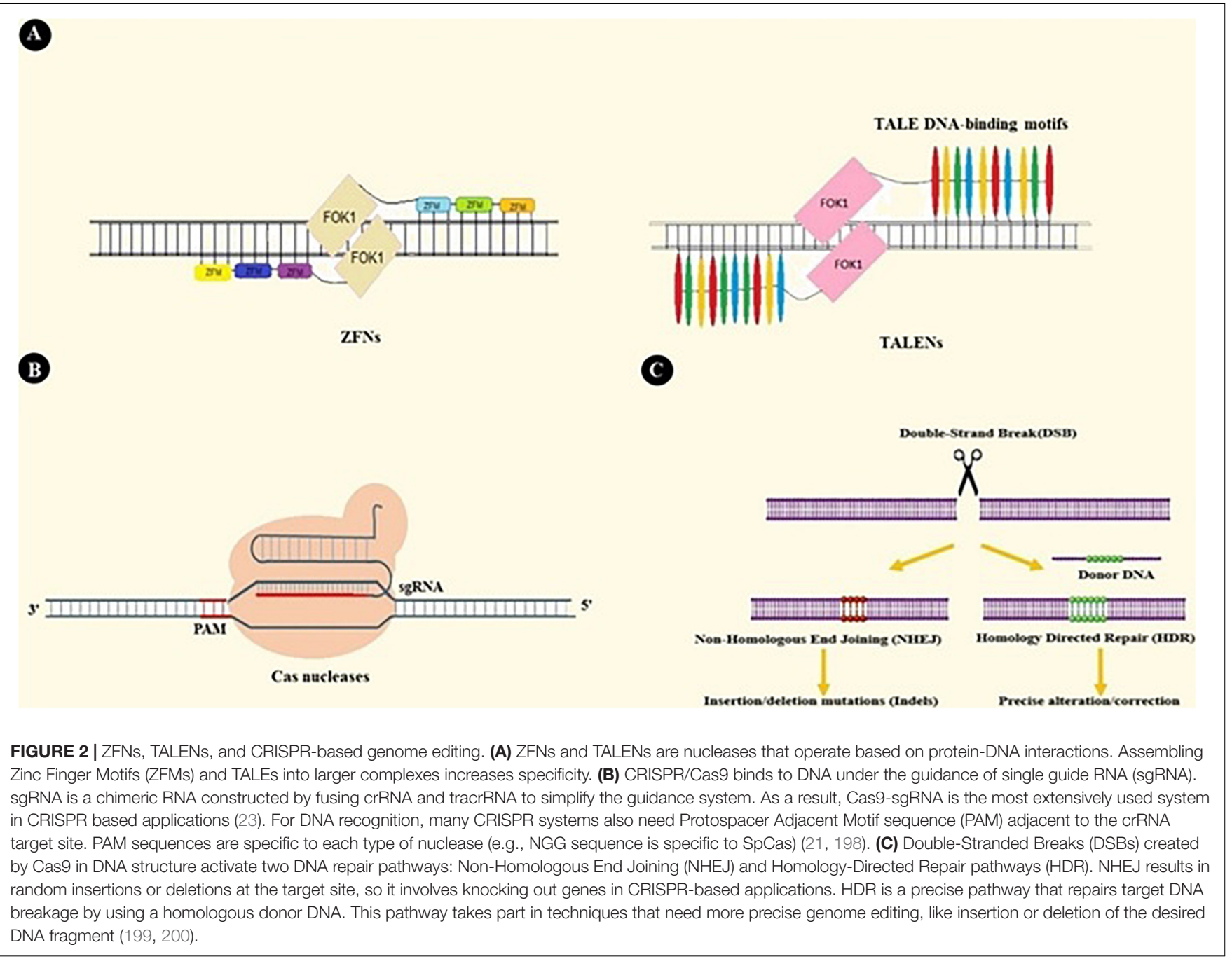

against these medications develops within 2 years. Huibin et al. proposed a molecular surgery using the CRISPR system to repair the mutated EGFR using the CRISPR/Cas9 nickase platform. Alternatively, this strategy would halt its activity by introducing a stop codon or an indel (random insertions and deletions) through HDR and NHEJ, respectively (32). This approach offers personalized gene therapy for disease-causing genetic abnormalities, which can be coupled with traditional therapeutic strategies, including surgeries and radiotherapy.

One of the main approaches to cancer cell therapy is knocking out genes responsible for inducing drug resistance. NFE2L2 gene [i.e., encodes Nuclear Factor Erythroid 2-Related Factor (NRF2)] is up-regulated under various conditions, such as oxidative or electrophilic stresses. These are consequences of chemotherapeutic drug administration as well. NRF2 targets numerous genes encoding GSH mediators, antioxidant proteins, and efflux pumps and induces cells' resistance against chemotherapy (33). Bialk et al. exploited CRISPR/Cas9 to knock out the NRF2 gene in chemo-resistant lung cancer cells. They reported restored effectiveness of anticancer drugs cisplatin, carboplatin, and vinorelbine post-gene editing (34). Therefore, the synergistic effects of combining gene edition and standard therapeutic options such as chemotherapy may address drug resistance-mediated refraction or relapse of the disease.

It is now known that epigenetic mechanisms play a critical role in different cancers' formation and progression (35). Recently, the CRISPR/Cas9 system has shed light on the underlying epigenetic irregularities and rendered researchers able to target these irregularities using the CRISPR/Cas9 platform. Wang et al. (36) targeted granulin (GRN), a liver cancer stem cell marker, epigenetically using the CRISPR/Cas9 system. The system consisted of C-terminus of the catalytically inactive dCas9 fused to three epigenetic suppressor domains: DNMT3a, histone 3 K27 methyltransferase EZH2, and heterochromatin binding suppressor KRAB. The group then designed gRNAs specific to the GRN promoter. Epigenetic targeting of GRN decreased tumor cell growth compared with the random gRNA control and dCas9 control groups (36-38), thus introducing a powerful epigenetic tool for oncogenes' inhibition.

Moreover, some viruses can cause malignant phenotypes in cells by inserting oncogenes into the cell genome. The CRISPR 

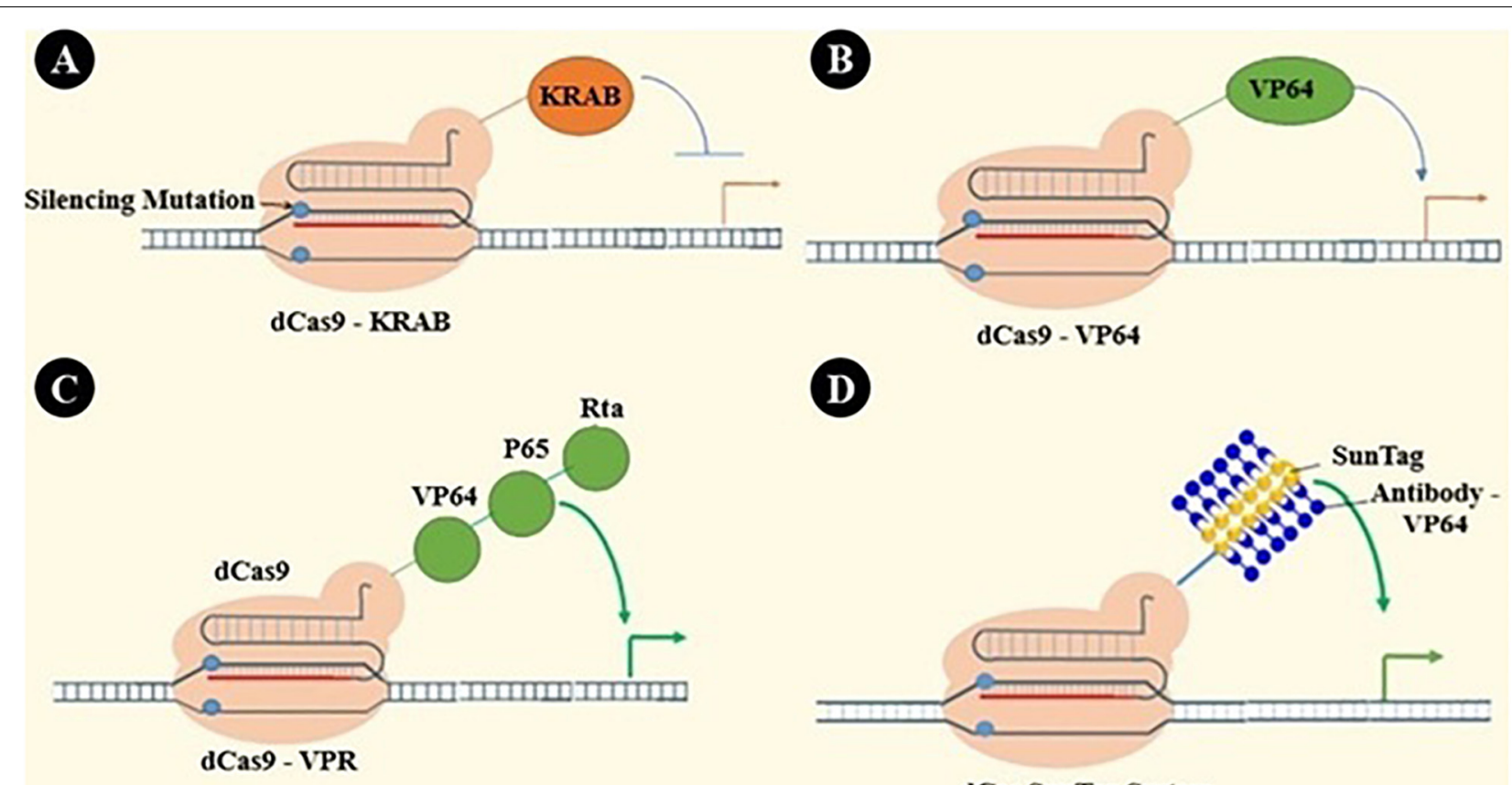

dCas SunTag System

E

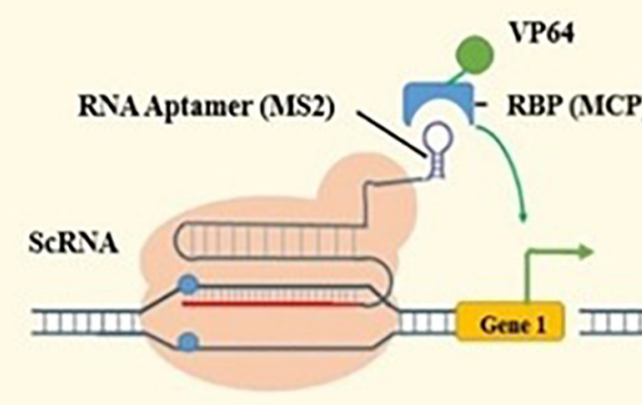

F

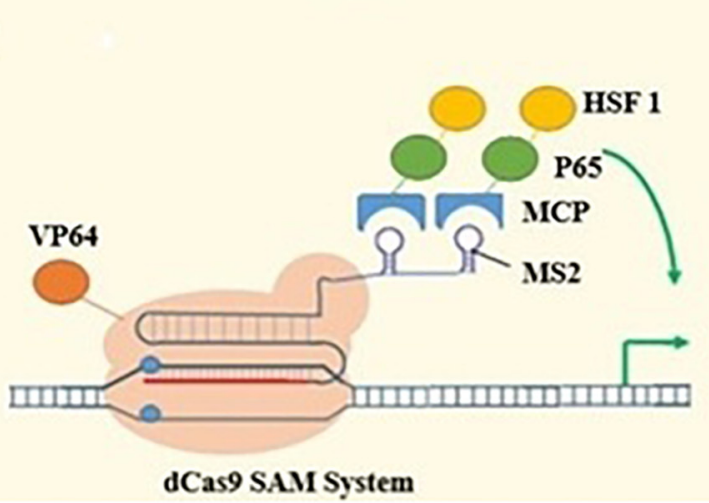

G

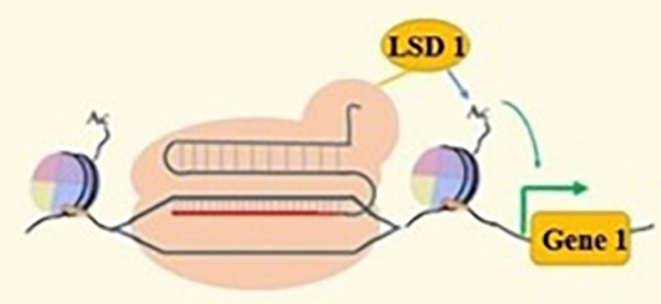

FIGURE 3 | dCas applications: beyond genome editing. (A) dCas9-KRAB has been engineered by fusing the KRAB transcription repressor domain to dCas9 (201). (B) VP64, as a transcription activator, has been fused to dCas9 to activate a specific gene's transcription (151, 152, 202, 203). (C) Recruiting multiple transcriptional activators shows synergistic effects that have led to engineering many systems, such as VPR by in-tandem fusing of both p65 and Rta to VP64 (204). (D) SunTag is a repeating protein-peptide array that recruits multiple antibody-fusion proteins. Protein domains, such as transcriptional activating or epigenetic modulating domains, can be recruited by antibody-mediated binding to SunTag, which is fused with dCas $(205,206)$. (E) RNA aptamers (e.g., MS2, com, PP7) can be fused with sgRNA to create a scaffold RNA (scRNA) that can recruit RNA-binding proteins (RBPs, e.g., MCP, Com, PCP). Fusing each RBP to the effector protein has enabled gene activation, repression, or even simultaneous activation and repression in one cell (207). (F) In the synergistic activation mediator (SAM) system, effectors are recruited by both dCas and scRNA (208). (G) Epigenetic modifying enzymes like P300 and LSD1 can be fused with dCas and alter cells' epigenomic features. These alterations were locus-specific epigenetic editing, including histone modifications and DNA methylations (205, 209, 210). 
system can be used against these virus-encoded oncogenes as well. The most famous example is the human papillomavirus, which is a primary causative agent for cervical cancer (39). Hsu et al. targeted E6 and E7 HPV-encoded oncogenes with CRISPR/Cas9 in patient-derived xenografts of HPV16 + anal tumors in immunodeficient mice and showed growth inhibition in tumor cells (40). CRISPR/Cas9 also induced proliferation arrest and decreased viral load in patient-derived cells suffering from Burkitt's lymphoma with latent Epstein-Barr virus infection (41). Based on these findings, CRISPR/Cas9 could be a promising therapeutic approach for the treatment of viral infectionrelated cancers.

As mentioned before, current CRISPR-based systems create DSBs which induce cellular DNA repair pathways, causing indels at the target locus. However, most diseases, including cancers, are caused by several point mutations, and there is a growing need for more efficient point mutation corrector tools. Therefore, novel Cas9 variants have been introduced that convert one base to another rather than creating DSBs. Accordingly, Komor et al. conjugated cytidine deaminase enzymes with dCas9 to convert a single base to another one (42). Their designed Base Editor (BE) was able to convert $\mathrm{C}$ to $\mathrm{T}$ to correct the p53 Tyr163Cys mutation that is associated with a variety of cancers (43). Following the same approach, Kuscu et al. introduced the CRISPR-STOP approach to create stop codons by converting single bases using BEs previously introduced by Komor et al. (44). Although the CRISPR-STOP approach has fewer potential sgRNAs for a target gene than wild type Cas9, introducing early stop codons is an efficient and safer gene-silencing approach.

\section{Prognostic and Predictive Biomarkers Identification via CRISPR/Cas}

Cancer biomarker discovery has emerged as an exciting area of research in recent years, mainly due to advancements in investigational screening tools for molecular level signatures, such as genomic-based alterations. Biomarkers, which are objectively measured and assessed features as an indicator of a biological status or process, can play a pivotal role in a patient's outcome. Prognostic and predictive biomarkers refer to biological characteristics that give information on the possible course of cancer and the patient's overall outcome irrespective of the therapy and predict the potential therapeutic outcome of a targeted treatment. The CRISPR/Cas platform can serve as a valuable tool to identify these biomarkers though assessing genetic or epigenetic changes within the tumor tissue $(45,46)$.

Cluster of Differentiation 44 (CD44) is a cell surface molecule that interacts with hyaluronic acid (HA) in the extracellular matrix. This interaction activates the mitogen-activated protein kinases (MAPK) and PI3 kinases/akt pathways in the act of oncogenic pathways $(47,48)$. Studies have revealed that CD44 binds to P-glycoprotein (P-gp), which is encoded by the $A B C B 1$ gene and acts as a drug efflux pump (49). Hence, its overexpression leads to resistance against chemotherapeutic drugs such as vinblastine, doxorubicin, and paclitaxel in osteosarcoma cells $(50,51)$. Xiaoa et al. used CRISPR/Cas9 to knock out CD44 in drug-resistant cell lines. P-gp levels were decreased as a consequence of CD44 knockout. CD44 knockout diminished resistance to doxorubicin in osteosarcoma cells (52). This study revealed that CD44 expression could serve as a predictor for overall survival and chemotherapy response and shed light on its role in tumor migration and tumorigenesis.

The Wnt-signaling pathway is considered as a pathway that initially drives cells' self-renewal in colorectal tumors (53). Mutations in this pathway induce the pathway to be constitutively active and provoke drug resistance. Furthermore, TIAM1, which encodes a guanine nucleotide exchange factor specific to Rac1, is a responsive gene to the Wnt signaling pathway and is overexpressed in human colon cancer (54). Izumi et al. generated xenograft mouse models with stable knockdown of TIAM1 using CRISPR/Cas9 and subsequently treated them with 5-fluorouracil (5-FU). TIAM1 knocked-down cells were more sensitive to 5-FU. Also, the tumor size and weight notably diminished compared to the controls. This study revealed the correlation between TIAM1 overexpression in CRC cells and also cancer-associated fibroblasts with drug resistance, serving as a predictive tool, and introduced this molecule as a potential therapeutic target to reverse drug resistance (55).

Qian et al. evaluated the effects of targeted DERARE methylation on leukemogenesis. Distal Element Multiple Retinoic Acid Response Element (DERARE) is a specific CisRegulatory Element (CRE) that maintains the homeostasis between self-renewal and multi-lineage differentiation. This effect is due to the regulation of Hoxb cluster genes in a methylation-dependent manner, which prevents leukemogenesis. The CRISPR/Cas9 epigenetic editing tool employed in this study was constructed by fusing the DNA methyltransferase $3 \mathrm{~A}$ (DNMT3A) catalytic domain to the $\mathrm{C}$ terminus of deactivated Cas9 (dCas9). In this fusion, there was a short linker enabling DNA methylation adjacent to the single guide RNA (sgRNA) binding site on human DERARE (56). The group infected human AML cell lines carrying the DNMT3A mutation via the lentiviral vector and observed a remarkable reduction in colony size and number in DNMT3A-dCAS9-treated AML cells (57). Their research proposes DNA methylation patterns on DERARE as a screening protocol for drug selection and implementing personalized therapeutic approaches.

MicroRNAs (miRNAs) are small, non-coding RNA molecules that play a role in cancer pathogenesis. There are different miRNAs implicated in all stages of cancer functioning as oncogenes or, conversely, as tumor suppressors. Hence assessing their upregulation and downregulation could be a potent tool to assess cancer progression (58). For example, a miRNA that is overexpressed in hepatocellular carcinoma cells is miR 3188. Zhou et al. demonstrated that this overexpression induces tumor formation and progression, resulting in poor clinical outcomes. Zhou and colleagues reported suppressed cell growth, colony formation, cell cycle progression, and increased apoptosis through CRISPR/Cas9-mediated miR-3188 knockout in cells (59), and proposed miR-3188 as a potent therapeutic target and also as a biomarker for early detection of HBV-related hepatocarcinogenesis among patients with a family history of HCC. 


\section{Identifying Novel Targets and Deciphering Drug Resistance via CRISPR/Cas}

CRISPR/Cas9 is a potent genome editing tool for discovering novel pathways and targets for cancer treatment and also unraveling the mechanisms responsible for inducing drug resistance and genes underlying these phenomena. CRISPR has an essential advantage over other genome editing technics (e.g., ZFNs and TALENs) because it can be easily retargeted to another locus in the genome by changing sgRNA design. Therefore, it is possible to knock out different genes with CRISPR/Cas9 in order to make explicit their role in cancer cell proliferation, survival, and metastasis, and also to dissect their contribution to developing drug resistance. Herein, we will review the results of some recent studies deploying this approach using the CRISPR/Cas9 genome -editing tool.

Feng et al. carried out an intervention using CRISPR/Cas9 to knock out $C D K 11 B$ in osteosarcoma cells. Inhibiting $C D K 11 B$ expression decreased cells' viability and their migratory and invasive activity. This study revealed the $C D K 11 B$ role in cancer pathogenesis and proposed its targeting as a potential approach in augmenting osteosarcoma patients' survival (60).

Urokinase Plasminogen Activator (uPA) binds to its receptor (uPAR), which is encoded by the PLAUR gene. Their interaction gives rise to extracellular matrix remodeling and subsequent cell adhesion, migration, proliferation, and survival via various signaling pathways $(61,62)$. Wang et al. revealed that uPAR knockout by CRISPR/Cas9 reduces resistance to chemotherapeutical drugs such as 5-FU, cisplatin, docetaxel, and doxorubicin (63). Hence, this proves the UPA/uPAR pathway as a potential target for intervention by other agents as well.

G-Protein-Coupled Receptor (GPCR) constitutes $40 \%$ of all drug targets approved by the FDA. GPRC5a, which encodes the G-Protein-Coupled Receptor family C, member 5, group A, also named retinoic acid-inducible 3 (RAI3), is overexpressed in a variety of cancers, including pancreas cancer (64, 65), which makes it a potential biomarker for early diagnosis of cancer. Liu et al. investigated the resistance of GPRC5a-knockout cells against commonly used chemotherapeutic drugs such as 5-FU, gemcitabine, and oxaliplatin. The group observed suppressed resistance in GPRC5a knockout cells compared with wild type cells using the EC50 (Concentration for $50 \%$ of maximal effect) assay (66). Targeting this signaling pathway, thus, appears to be an exciting area of further research.

$K R A S$ is the most frequently mutated proto-oncogene in cancer cells, and its pharmacological targeting has remained challenging in cancer therapy (67). Since activating mutations in KRAS are a hallmark of pancreatic ductal adenocarcinoma (PDAC), CRISPR/Cas was employed in a study to model complete KRAS inhibition and predict resistance mechanisms in a subset of human and mouse PDAC cells. This study showed the merit of KRAS-directed therapies in reducing in vitro proliferation and in vivo tumorigenic growth and revealed KRAS' role in balancing proliferation and metastasis in tumor cells. This study also recommended PI3K pathway activation as a potential resistance mechanism in KRAS knocked-out cells and suggested simultaneous PI3K and KRAS inhibition as a therapeutic strategy for PDAC (68).

The N-Myc oncoprotein, which is overexpressed in a fraction of different types of prostate cancers (69-71), possesses identified functions in tumor progression $(70,72)$. Yin et al. reported that the $\mathrm{N}-\mathrm{Myc}$-regulated DNA Damage Response (DDR) pathway (N-Myc/miR-421/ATM) is associated with tumor progression and hormonal therapy resistance, such as enzalutamide resistance. N-Myc overexpression cooperates with EZH2 to suppress miR-421. This suppression leads to ATM up-regulation, resulting in enzalutamide resistance. Yin and colleagues knocked out ATM via the CRISPR/Cas9 genome editing tool and detected re-sensitized prostate cancer cells (73).

Apolipoprotein B mRNA editing the enzyme catalytic polypeptide-like (APOBEC) enzyme family is involved in genetic instability and heterogeneity. APOBEC enzyme family derived DNA mutagenesis patterns have been detected in different types of cancers (74), including breast (75), bladder, cervix, head and neck, lung cancers (76), and gliomas (77). Schmitt et al. investigated the role of APOBEC 3B in glioma cells' resistance to temozolomide by determining the caspase 3/7 activity. They knocked down APOBEC3B using CRISPER/Cas9 and observed higher sensitivity toward temozolomide relative to the control cells (77).

miR-21 is a miRNA overexpressed in cancers. Huo et al. hampered its expression via indels introduced by CRISPR/Cas9. The group reported the inhibition of cell proliferation, migration, and invasion in ovarian cancer cells. The hampered expression of miR-21 was associated with higher drug sensitivity and decreased Epithelial to Mesenchymal Transition (EMT), revealing its role in tumor metastasis (78). The latter plays a critical role in metastasis and chemoresistance (79).

\section{CRISPR/Cas Genome-Wide Knockout Screening}

CRISPR/Cas genome-scale knockout screening refers to the process of sgRNA-mediated disruption of genes' functionalities aiming to discover novel genes and pathways underlying various phenotypes and biological processes, including tumorigenesis and drug resistance in cancer, and also their potential as therapeutic targets $(80,81)$. Numerous studies have employed genome-wide knockout libraries (GeCKO) which contain a multitude of sgRNAs against a defined set of genes involved in cancer. sgRNAs in the cells affect their competence for viability during the proliferation. The enrichment or depletion of these sgRNAs thereupon reveals the genes responsible for the cognate phenotype (82-88).

Among the pioneering groups to both develop and implement GeCKO $(15,89)$, Shalem et al. unraveled novel genes responsible for the development of resistance to Vemurafenib (PLX) (i.e., a BRAF protein kinase inhibitor) in melanoma. Cells were transduced with a pool of lentiviruses each carrying Cas9 and a sgRNA. Thereafter, cells underwent PLX selection. The enriched sgRNAs in viable cells were then sequenced and their target genes, whose loss of function contributed to the development of resistance, were revealed (15). Decoding the mechanisms 
underlying resistance to drugs can introduce novel predictive biomarkers and even novel targets.

In a similar study, Manguso et al. unraveled the mechanisms underlying the resistance to PD-1/PD-L1 inhibition treatment using a library of lenti-vectors with sgRNAs targeting 2368 murine genes. This study revealed Ptpn2 as a potential target to revert PD-L1 immunotherapy resistance since its knockout was associated with increased sensitivity to immunotherapy (90). IRF4, STAT3, SOS1, and GRB2 genes' knockout in ALK + ALCL cells also attenuated PD-L1 expression and undermined PD-L1 mediated $\mathrm{T}$ cells and NK cells suppression (91).

Genes responsible for the resistance to Bortezomib (BTZ) in Multiple Myeloma have been determined by conducting a genome-scale positive selection assay. Multiple myeloma cells transduced with sgRNA-carrying lentiviruses were cultured in the presence of BTZ at its lethal dose. The inactivated genes in survived cells were identified based on the enriched sgRNAs sequencing, and proteasome regulatory subunit PSMC6 was proven to be the only gene that granted resistance to BTZ reproducibly. Therefore, PSMC6 emerged to be a promising predictive biomarker and also a novel target (92).

This strategy has also been employed to discover the mechanism of action of Immunomodulatory imide drugs (IMiDs), including mediating pomalidomide and lenalidomide. Liu et al. explored the mechanism responsible for the susceptibility of multiple myeloma cell lines to IMiDs by loss-of-function genome-wide screening. The team found CRBN regulation mediated by CSNs as the major factor determining multiple myeloma cells' sensitivity to IMiDs (93).

CRISPR genome-wide screening has also proven to be beneficial in novel target identification. A dropout screen on AML cell lines revealed that the sgRNA-mediated knockout of KAT2A hampers AML cell lines' growth. The same outcome was observed when AML cell lines were treated with MB-3, a KAT2A inhibitor. Therefore, since KAT2A is not an essential gene for hematopoietic progenitor cells, its inhibition is proposed as a novel anti-AML therapeutic strategy and MB-3 as a potential medication for AML (94).

Another genome-wide negative selection screen on AML employed mouse lentivirus-based GeCKO v2 library. Among the genes dispensable for human hematopoiesis, the mRNA de-capping enzyme scavenger (DCPS) appeared to be essential for AML cell survival. Its inhibitor, RG3039, exhibited antileukemia effects in human AML xenograft models, sparking its combination with other drugs as a potential therapeutic approach (95).

CRISPR/Cas has surpassed RNA interference (RNAi) technology in building genome-wide knockout libraries due to having a lower rate of off-targets and also introducing lossof-function mutations into the gene's sequence as opposed to RNAi which only yields the gene's partial suppression in the majority of cases $(15,81,96)$. However, CRISPR/Cas imposes some limitations; first, during drop-out screenings, CRISPR/Cas has shown conditional false-positive results in cancers with aneuploidy. Second, genomic regions with multiple copy numbers, including non-expressed genes, are subject to excessive double-strand breaks (DSBs). DSBs in turn lead to substantial
DNA damage and subsequently induce apoptosis. Hence, sgRNAs targeting non-expressed genes should be excluded from the libraries. Third, sgRNAs are conventionally designed to target 5 ' exon. However, false-negative results have been attributed to genes with initiation points in other exons as well, signifying the effect of sgRNA positioning in the accuracy of the outcomes (97).

\section{CRISPR AND IMMUNOTHERAPY}

It has been established that evading immune destruction is one of the hallmarks of cancer. Tumor cells can inhibit immune effector cells or cause immune tolerance through the secretion of extrinsic factors affecting the tumor microenvironment (TME) (98). Among all the immune system members present at TME, macrophages and T cells are the most distorted. Tumorassociated macrophages (TAMs) support tumorigenesis and metastasis and inhibit antitumor responses by releasing EGF, IL-6, TNF, MMPs, VEGFA, TGF- $\beta$, IL-10, and PD-L1 $(98,99)$. In addition, $\mathrm{T}$ cells' anti-tumor activity and metabolic state is disrupted by the immune-modulatory cytokines present in the TME and the immune checkpoint inhibitors such as PD1 and CTLA-4 $(99,100)$. Accordingly, a study by Chung et al. on 11 breast cancer cases revealed that the presence of M2 macrophages in the TME was correlated with T cell exhaustion (101). Therefore, the state of TME strongly affects the patients' prognosis. Hence, identifying the mechanisms underlying the tumorigenic characteristics of interactions between immune suppressive cells and tumors can reveal novel therapeutic targets for developing antagonists, such as mAbs, and immunomodulatory drugs intervention.

Another approach is to fortify already existing immune responses or develop new ones through bypassing their dependence on the robust and intact immune system, which, as explained before, had transformed into a non-functional state (102). Adoptive T cell immunotherapy of cancer has recently proven its potential in numerous clinical trials, yet it still suffers from various predicaments (103).

CRISPR/Cas-mediated genetic manipulation has strived to address some of the challenges mentioned above regarding immune system misfunctioning from various prospects, some of which are discussed below.

\section{Novel Immune System-Tumor Interplay Identification via CRISPR/Cas}

TME is composed of tumor cells, stromal cells, and immune cells, and the interaction among these cells affects tumor progression. Stimulants present in the TME, such as cytokines, chemokines, and growth factors, determine the polarization of macrophages and their differentiation toward M1 or M2 subtypes. M1 macrophages are able to: (i) release pro-inflammatory cytokines such as IL-12, IFN gamma, IL-1, IL-23, and iNOS; (ii) reeducate the DC and CD4 + T cells; and (iii) activate CD8 + T cells and, as a result, promote an immune response against the tumor and prevent tumor progression. In contrast, M2 macrophages and TAM increase angiogenesis and formation of tumor-associated 
fibroblasts. These cells attenuate immune responses in the TME and increase tumor progression (104-107).

Various agents that participate in the interaction between tumor cells and M2 macrophages have been targeted using the CRISPR/Cas9 system. These targets include: (I) macrophage Signal Regulatory Protein a (SIRPa). The crosstalk between SIRP-a on macrophages and CD47 receptors on tumor cells prevents phagocytosis of cancerous cells via the "Don't eat me" signal. Turning off this signaling by knocking out the SIRP- $\alpha$ using CRISPR/Cas9 enables phagocytosis of cancer cells (108); (II) Kindlin2, this protein is another therapeutic target that increases the secretion of Cancer Stimulating Factor1 (CSF1) from tumor cells. Moreover, Kindlin2 induces chemotaxis of macrophages to the TME, which subsequently constitutes the dominant population. Ablating Kindlin2 expression using CRISPR/Cas9 technology inhibited invasion and migration of tumor cells without affecting their proliferation rate (109); (III) Osteopontin (OPN) glycophosphoprotein in tumor cells increases the recruitment of M2 macrophages. Therefore, it has been targeted in cancer treatment. OPN knockout in tumor cells by CRISPR/Cas9 decreases the chemotaxis of macrophages and increases the sensitivity of cancer cells to CD8 $+\mathrm{T}$ cells cytotoxicity (110); (IV) Lysosome Associated Membrane Protein Type 2A (LAMP2a) is another agent expression which is increased by tumor cells in TAMs. LAMP2a inactivation using CRISPR/Cas9 reduces TAM activation and prevents the suppression of the immune system and decreases tumor growth (111); (V) IL-8 released by macrophages increases tumor growth and metastasis. It has been revealed that knock out of the IL- 8 receptor, CXCR2, by CRISPR/Cas9 in triplenegative breast cancer cell lines reduces the progression of the tumor (112); and (VI) Tumor-Secreted Protein S (Pros1) is the best-studied ligand of Tyro3/Axl/Mer (TAM) receptor tyrosine kinases, and its CRISPR-based deletion inhibits M2 polarization, leading to heightened immune infiltration and reduced tumor viability (113).

Theoretically, many other potential targets can affect macrophage polarization toward the M2 phenotype and their immunosuppressive features. Therefore, this could be a hot topic for future investigations not only in treating cancer using CRISPR technology but also in finding suitable targets for pharmacological drugs or monoclonal antibodies.

\section{Novel Cellular Immunotherapy via CRISPR/Cas}

Immune cell therapy has emerged as a novel approach after traditional pharmaceuticals such as small molecules and biopharmaceuticals, like therapeutic proteins, including mAbs (114). Due to an extensive TCR repertoire and their ability to distinguish themselves from non-self-epitopes produced during tumorigenesis, $\mathrm{T}$ lymphocytes play a pivotal role in tumor surveillance and cancer eradication. Thus, attempts have been made to produce, guide, or enhance cellular immunotherapy against cancer over the past decades. T cellbased immunotherapy is attributed to the implementation of ex vivo manipulated $\mathrm{T}$ lymphocytes aiming to eliminate tumors with TCR-engineered T lymphocytes and Chimeric Antigen Receptors T cells (CAR T Cells) as its main strategies $(83,102)$. In the former, patient's $\mathrm{T}$ lymphocytes are transfected to express a transgenic TCR derived from other patients or animal models with specificity against a Tumor-Associated Antigen (TAA), and the latter exploits chimeric receptors with their antigen recognizing domains mostly acquired from antibodies (115).

\section{Universal CAR T Cells Production via CRISPR/Cas}

Despite significant breakthroughs in CAR T Cells' application in hematologic malignancies elimination, there are still considerable barriers in the application of CAR T cell therapy, specifically against solid tumors, and also throughout its laborious manufacturing process (116). In CAR T cell therapy, $\mathrm{T}$ cells can be derived from patients (autologous) or an allogeneic donor. Using autologous T cells is a time-consuming process and largely depends on the quality and quantity of autologous $\mathrm{T}$ cells harvested from the patient. These issues are also coupled with the expense of manufacturing autologous T cells $(117,118)$. One of the substantial barriers in using allogeneic T cells is the presence of endogenous MHC class I and TCR on donor's T lymphocytes, which cause alloreactivity and graft-versus-host disease (GVHD), respectively. The former is dependent on the TCR repertoire of the recipient $(119,120)$.

To overcome the aforementioned HLA barriers in the implementation of third-party donors-derived T lymphocytes, Poirot et al. were first able to knock out endogenous TCR by TALEN-mediated disruption of $\mathrm{T}$ cell receptor alpha constant chain (TRAC) in lentiviral-transduced CD19 CAR T cells (121). This platform was further entered into clinical studies on two infants with relapsed refractory CD19 + B cell ALL (122). "Universal CAR T cells" are now being manufactured by knocking out TCR and HLA-I in allogeneic T cells (123). Furthermore, Eyquem et al. have exploited CRISPR/Cas9 to insert the CAR gene and remove the TCR gene concurrently by introducing the CAR gene into the TRAC locus. They observed a regular CAR expression in $\mathrm{T}$ cells, increased potency of $\mathrm{T}$ cells, and decreased terminal differentiation and exhaustion in the mouse model of AML (124).

CRISPR/Cas9 technology was further used to couple allogenic CAR $\mathrm{T}$ cells and checkpoint pathway disruption. In a study by Ren et al. CRISPR/Cas9 technology was employed to knock out PDCD1, TRAC, and beta-2-microglobulin ( $\beta 2 \mathrm{M})$, which encodes the accessory chain of MHC class I in CD19 or PSCA CAR T cells. These $\mathrm{T}$ cells exhibited robust antitumor activity and did not induce GVHD in the leukemia mice model (125). In a similar study on EGFRvIII-targeted CAR T cells and their triple geneedited CAR, T cells displayed an enhanced profile in preclinical glioblastoma models (126).

Of $\mathrm{T}$ cell-based immunotherapies, the necessity of TCR knockout is not confined to CAR $T$ cell therapy. In TCRengineered T cells, the $\alpha$ and $\beta$ chains of endogenous TCR are shown to pair with the transgenic TCR $\alpha$ and $\beta$ chains. This mispairing disrupts efficient $\mathrm{T}$ cell redirection toward the targeted antigen and also poses the risk of novel autoreactivity. Moreover, competition of these four chains for binding to the CD3 complex hampers the translocation and, consequently, 
the sufficient display of transgenic TCR on the cell surface. These are among the culprits underlying the poor performance of TCR-engineered $\mathrm{T}$ cells in clinical studies $(127,128)$. In a pioneering study by Provasi et al., ZFNs were designed to obstruct endogenous TCR $\beta$ and $\alpha$ chain genes expression and a higher level of cell surface exogenous TCR display alongside superior specificity was reported (129). CRISPR/Cas-mediated disruption of surface TCR expression in TCR-engineered T cells has paved its way into clinical studies, and will be discussed in the following sections (130).

\section{Immune Checkpoint Inhibition via CRISPR/Cas}

Engineered T cells' activity is susceptible to be impeded via natural immune checkpoint regulators. Thus, identification of these immune checkpoint regulators, such as programmed cell death protein 1 (PD-1), cytotoxic T-lymphocyte-associated protein 4 (CTLA-4), and other inhibitory signaling, has created a new vision for cancer immunotherapy $(131,132)$. Overexpression of immune checkpoint regulators and up-regulation of their cognate inhibitory ligands (e.g., PDL1 and CTLA4 ligand) in the TME may limit TCR-engineered and CAR T cell persistence and function. Accordingly, this will lead to impaired clinical outcomes of this strategy (15). The CRISPR/Cas9-based editing could be used to ablate PD-1 and CTLA- 4 in order to increase the efficiency of T cell-based immunotherapy (125).

Gene knock-out of PD-1 in Car $\mathrm{T}$ cells using CRISPR technology was first applied by Su et al., and resulted in enhanced cytotoxicity without affecting $T$ cells viability (133). Since then, several groups reported similar results while administering different methods. $\mathrm{Hu}$ et al. used CRISPR/Cas9 against the PDCD1 gene, accompanied by anti CD133 CAR insertion into the genome using the piggyback transposon system. They reported increased $\mathrm{T}$ cell proliferation and cytokine secretion (134). In another study, a ground-breaking one-step system named knock-in and immune-checkpoint knockout (KIKO CAR-T cell) was developed, which relied on the cpfl system to mediate simultaneous knock-in of two different CARs and knockout of PD-1 and TRAC. Elevated cytokine production and cytotoxicity and decreased levels of exhaustion markers were reported (135). PD-1 knockout CAR T cells were also assessed against glioblastoma, hepatocellular, and K562 tumor cell lines and demonstrated enhanced anti-tumor activity, reduced exhaustion, and augmented killing power in Car T cells (136-138). CRISPR/Cas9-mediated PD-1 knockout in T cells is now under clinical evaluation in a phase one trial on metastatic non-small-cell lung cancer (139).

Zhang et al. successfully generated lymphocyte activating gene-3 (LAG-3) knock-out CAR T cells using CRISPR/Cas9. They reported no significant viability or immunophenotypic changes in cultured CAR $\mathrm{T}$ cells in vitro. However, LAG-3 knockout CAR T cells possessed more vigorous antigen-specific anti-tumor activity in a xenograft mouse model (140).

The Fas receptor CD95 binds to its ligand, which is overexpressed on tumor cells and induces T cells' apoptosis and loss of function (141). In order to increase resistance to Fasmediated apoptosis, Ren and colleagues generated allogeneic universal CAR T cells via double knockout of endogenous
TCR and HLA class I (HLA-I). Afterward, they disrupted the Fas receptor, PD1, and CTLA-4 in the same way and finally accomplished quadruple gene disruption in $\mathrm{T}$ cells with the one-shot system. Also, generating universal allogeneic $\mathrm{T}$ cells with multiple negative regulators (i.e., PD-1 and CD95/Fas death receptor) knocked out has been demonstrated (142).

\section{CAR T Cell Functionality Augmentation via CRISPR/Cas}

Using CRISPR/Cas9 to knock out the Granulocyte-Macrophage Colony Stimulating Factor (GM-CSF) gene in CAR T cells increased their antitumor activity and survival. Knocking out the GM-CSF gene not only increased CAR T cell activity but also decreased neuroinflammation and the probability of CRS (143).

It is known that CD7 targeting CAR $\mathrm{T}$ cells can destroy each other by targeting CD7 markers present on themselves, an action termed fratricidal activity. Silva et al. indicated that knocking out CD7 in CAR T cells using CRISPR/Cas9 prevents fratricidal activity followed by CAR $\mathrm{T}$ cell immunotherapies (144). Moreover, knocking out CD7 and TRAC in CAR T cells increased the efficacy in the treatment of $\mathrm{T}$ cell acute lymphoblastic leukemia (T ALL) (145).

Jung et al. used CRISPR/Cas9 to knock out Diacylglycerol Kinase (DGK) in CAR T cells. DGK is an enzyme that metabolizes diacylglycerol to phosphatidic acid (PA), knocking out the DGK gene increases CD3 signaling and improves $\mathrm{T}$ cell function by boosting TCR signaling (146).

Studies have confirmed that knocking down TET2, a tumor suppressor gene, leads to epigenetic and phenotypic alterations in T cells, which can improve clinical results (147). Fraietta et al. showed that using CRISPR/Cas9 to knock out the CD19 CAR gene into the TET2 locus promotes anti CD19 CAR T cell activity (147).

\section{CRISPR/Cas and Oncolytic Virotherapy}

Oncolytic viruses (OVs) possess a higher tendency to infect and replicate within cancerous cells than normal cells. Based on the promise of naturally occurring OVs, their further genetic manipulation has turned into an up-and-coming strategy (148). These manipulations mainly focus on increasing their tumor-selectivity, tumor tropism, and therapeutic efficacy and decreasing their off-tumor toxicity and pathogenicity against non-neoplastic cells. They mainly stem from the normal cells' ability to develop intracellular anti-virus defense mechanisms which are mostly accompanied by the host cell's apoptosis. In neoplastic cells, on the other hand, anti-apoptotic molecules are overexpressed and programmed cell death is obscured. Hence, the anti-virus immune-compromised cells turn into a suitable place for OVs to replicate and subsequently to burst the cell itself and to spread viral particles' locally among other cancerous cells $(149,150)$. The first and only $\mathrm{OV}$ to attain FDA approval is T-VEC (IMLYGIC ${ }^{\circledR}$ ) with an indication for advanced melanoma (151). T-VCE is an attenuated Herpes Simplex Virus 1 (HSV-1) with decreased pathogenicity and increased tumor selectivity. It is also engineered to release GM-CSF and to enhance MHC-mediated antigen presentation (151). The potential of $\mathrm{OV}$ in cancer 
therapy and its genetic manipulation by strategies except CRISPR/Cas have been extensively reviewed elsewhere (152155). Here, the focus is on the application of CRISPR/Cas in oncolytic virotherapy.

Genetic manipulation of OVs with large genomes, such as HSV, Adenovirus (Adv), and Vaccinia Virus (VACV), by traditional techniques is laborious and has low efficiency. CRISPR/Cas, on the other hand, expedites recombinant OV generation. It simplifies the process of deletion and insertion compared to the traditional strategies (156).

To achieve tumor selectivity and oncolytic efficacy, HSV1 genes have been repeatedly subject to CRISPR/Cas-mediated knockout both through NHEJ (156) and HDR-mediated replacement with foreign genes (156-158). CRISPR/Cas has also been able to mediate multiple knockouts in HSV-1 (158). Various approaches have been implemented to further enhance the knockout efficiency, including enrichment via selectable markers, and also the incorporation of Scr7, an NHEJ inhibitor, to enhance HDR/NHEJ ratio in HSV-1 $(157,158)$. Collectively, all of these studies revealed low off-target activity of the CRISPR/Cas platform (156-158). HDR-mediated knock-in has demonstrated to be feasible and more efficient relative to mere homologous recombination in HSV-1 in several studies (156-158).

VACV is another OV with great therapeutic potential with numerous complete and undergoing clinical trials (159). CRISPR/Cas brought about simultaneous knockout of viral N1L, which plays a pivotal role in VACV virulence and host immune response modulation to VACV and TRP2, which is a TAA, inside the N1L locus (160). Theoretically, delivery of TRP2 to the tumor and induction of adaptive immune response can promote VACV into a cancer therapeutic viral vaccine. CRISPR/Cas-mediated simultaneous double knockout of two immune-regulatory genes, N1L and A46R, has also been reported and is presumed to enhance VACV immune response induction. Accordingly, it can be concluded that sgRNA-guided Cas9 can concomitantly target multiple sites on the VACV genome (160).

CRISPR/Cas system was used to exert indels into the EGFP gene in a recombinant adenoviral vector. The mutations' inheritance to the next generations alongside its safety in terms of off-tumor activity were observed as well (156).

Plus, the CRISPR/Cas platform can be utilized to equip OVs with immune-stimulants or anti-tumoral agents. In a study by Cai and colleagues, oncolytic Human Simplex Virus 2 (oHSV-2) was armed with murine IL-15 via CRISPR/Cas9. The continuous intra-tumoral expression of IL-15 enhanced T cells anti-tumor response and also reduced the tumor mass. Incorporation of immune-modulators within the genome of OVs obviates the need for their systematic administration. Due to the local release of the immune-stimulants, this strategy subsequently reduces the risk of systematic side effects (161).

In addition, OVs can join their gene therapy delivery potential and their intrinsic anti-tumor activity to elicit a synergistic therapeutic response. Following the primary finding that CRISPR/Cas-mediated RAS knockout results in tumor regression in Rhabdomyosarcoma, Phelps and colleagues developed a CRISPR/Cas-harboring recombinant Myxoma Virus. This OV carried a spCas9-2A-Csy4 cassette followed by two NRAS-targeting sgRNAs. Csy4 ribonuclease was incorporated to split the sgRNAs from the 3' end of mRNA molecules. A significant although not sustainable reduction in xenograft tumor growth was reported and further enhancing the tumor-specificity of this OV is recommended (162).

\section{CRISPR/Cas IN CANCER CLINICAL TRIALS}

In early 2018, the University of Pennsylvania, in collaboration with Tmunity, launched the first-in-human phase 1 CRISPR gene editing trial on NY-ESO-1 targeting TCR-engineered T cells in melanoma and myeloma patients. These T cells lacked TRAC, TRBC, and PDCD1 genes expression through CRISPR/Cas9mediated editing. This study confirmed the safety of multiplex CRISPR-Cas9 editing of the human genome since neither of the subjects displayed cytokine release syndrome (CRS) or other side effects. Additionally, no rejection of transferred $\mathrm{T}$ cells due to pre-existing immunity against Cas9 among individuals was observed, further approving the potential of this technology in cancer treatment (130).

While Allogene Therapeutics and Cellectis utilize TALEN to produce TCR and MHC knockout universal CARs in their clinical trials (NCT04093596, NCT04106076, NCT03190278 to name a few), in July 2019, CRISPR Therapeutics initiated its first clinical trial evaluating CTX110 (NCT04035434). CTX110 are universal CD19-directed CAR T cells developed through using CRISPR/Cas9 to insert CAR into the TRAC locus, resulting in endogenous TCR disruption and also knockout of B2M and thus MHC1 for Refractory/Relapsed B cell malignancies.

In a clinical trial by Baylor College of Medicine (NCT03690011), T cells underwent endogenous CD7 knockout through CRISPR/Cas9 technology prior to receiving CD7targeting CARs in order to avoid fratricidal activity. This study, which is designed for high-risk T-cell malignancies, is yet to recruit patients.

The Clinical application of the CRISPR/Cas9 platform is not confined to engineering cellular-based therapeutics. In a study initiated in mid-2018 (NCT03606486), the University of Washington developed a minimally invasive test to detect ovarian cancer through screening cervix pap smear samples for tumor-associated mutations in TP53 (i.e., the most common mutated gene in ovarian cancer). The team employed CRISPR-Duplex sequencing, which combines ultraaccurate Duplex Sequencing with CRISPR/Cas9 excision of target regions, leading to enrichment through size selection before sequencing library preparation (163). In another study in the Children's Research Institute (NCT03332030), an induced Pluripotent Stem Cell (iPSC) bank was established for patients with Neurofibromatosis type 1 (NF1) phenotype. After that, CRISPR/Cas9 technology was utilized to develop isogenic NF1 wild-type $(\mathrm{NF} 1+/+)$, NF1 heterozygous (NF1 \pm ), and NF1 homozygous (NF1-/-) iPSC lines from individual patients. These iPSC lines are then differentiated to central nervous system tumor-relevant cells. They are screened to identify the drugs that have the potential to reverse or alleviate the disease phenotypes. 
a

(b)

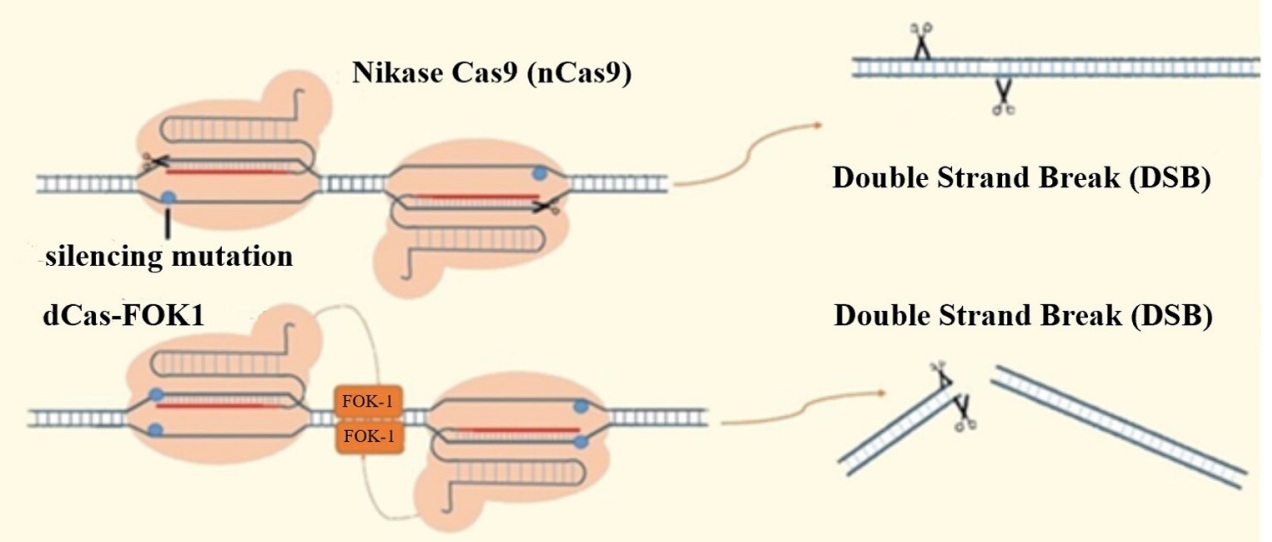

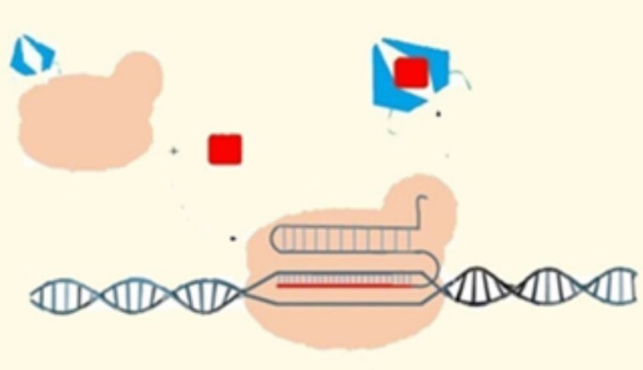

Intein- Cas

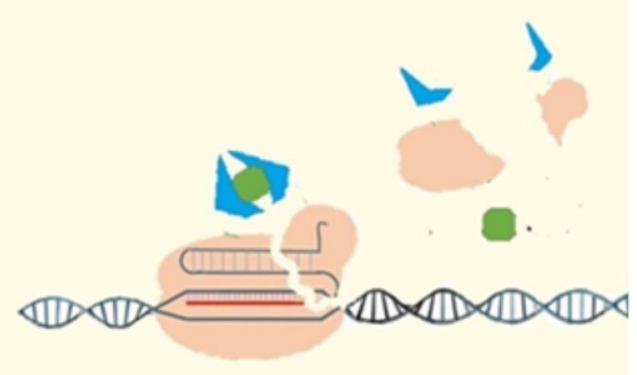

Inducible Split-Cas

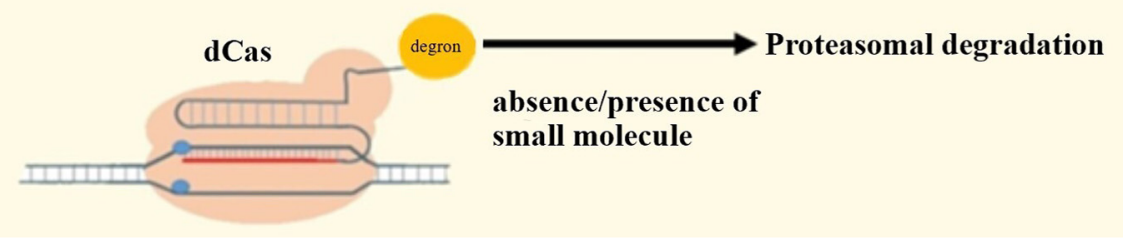

FIGURE 4 | Some strategies to solve CRISPR system limitations. (a) Systems that need dimerization to cleave both strands: 1. dCas-FOK1 2. nCas9. (b) Cas9 variants' activity can be switched on and off by cell-permeable molecules: (1) Intein-Cas9 is activated by excision of the intein bound to a specific position in Cas9. A cell-permeable small molecule induces this excision $(189,211)$. (2) Split-Cas9 is a Cas9 molecule that has been split into two fragments, and these two can be dimerized via drug-binding dimerization domains and a cell-permeable drug (212). (3) Degron-Cas9 is formed by binding a destabilizing domain (degron) to Cas9 protein. Previous studies have introduced a different type of degron that can bind to the protein of interest and decrease the stability of that protein in the presence or absence of specific small molecules. Degron domains can also be fused to the RBP-effector complex to regulate its stability and activity of the CRISPR system as a result $(190,213-216)$.

\section{CRISPR: LIMITATIONS TO SOLVE}

CRISPR immune-related adverse effects pose constraints in its therapeutic applications. The delivery system, Cas protein, and sgRNA can all evoke the host innate and acquired immune system. Pre-existing antibodies against SaCas9 and SpCas9 were found in 78 and 58\% of donors, respectively. Likewise, antiSaCas9 and anti-SpCas9 $\mathrm{T}$ cells were found in 78 and $67 \%$ of donors (164). Furthermore, pattern recognition receptors might recognize the secondary structure of sgRNAs and initiate an immune response (78). Further studies are required to ascertain whether or not these immune reactions could restrict the clinical potential of this platform (4).

The CRISPR/Cas9 system creates double-strand breaks in the genome, which can induce p53-mediated apoptosis in transfected cells and reduce cell viability. Wildtype p53 cells may die, whereas mutant p53 cells can be selected as a result of Darwinian selection-like evolution. Therefore, P53 inhibition can improve the efficacy of genome editing. In order to reduce the risk concerning p53 mutation in cells in cell replacement therapies, p53 function should be monitored (165, 166). 
At the CRISPR target site, significant on-target mutagenesis, such as deletions and genomic rearrangements, were reported, which may have pathogenic effects (167). Incorporating gene encoding chimeric suicide receptors alongside the platform can induce drug-dependent apoptosis. These receptors bind to a drug by their extracellular domain and use a caspase 9 endodomain as an effector (168). This strategy and similar common strategies in CAR T cell therapy using the suicide gene increases safety in using CRISPR mediated genome-edited cells in case of unpredicted adverse effects (169).

Off-target effects (OTEs) are still the primary limiting concern in the application of genome editing in clinical trials (170). Designing appropriate gRNA, selecting more specific nucleases, and restricting the exposure time to active nucleases are three primary strategies in reducing OTEs.

Appropriate gRNA design can significantly reduce OTEs and many gRNA design software tools that predict the probability of off-target cutting, such as elevation, azimuth, and benching (171). Truncating $5^{\prime}$ end of gRNAs and chemical modification of crRNA sites for impairing hybridization to off-target sequences are possible changes implicated in gRNA that improve specificity $(172,173)$.

The choice of nuclease is another famous gate through which enhanced specificity can be achieved. Cpf1 and fnCas9 have been reported to have higher specificity than SpCas9 $(174,175)$. Changing amino acid residues for reducing OTEs has led to engineering high fidelity Cas9 proteins such as SpCas9-HF1, eSpCas9, and HypaCas9. The mechanism of action of these altered Cas9 proteins might be a change in nuclease domain activation pattern ( $\mathrm{HNH}$ domain) or change in the strength of binding to target DNA (176-178). In an attempt to construct a SpCas9 that can recognize a broad range of PAM sequences, several Cas proteins with reduced OTEs were created $(179,180)$. Another form of nucleases was developed by fusing dCas to the FOK1 cleavage domain that generates a chimeric protein that needs dimerization for DNA cleavage. Since two closely located sequences' recognition is needed through two distinct sgRNAs, this approach theoretically increases specificity (181). There is a similar strategy using nickase Cas9 that has a mutation in one of two nuclease domains of Cas9 protein. As a result, each nCas9, which is guided with a separate sgRNA, cleaves only one strand of DNA (Figure 4A) (182).

Limiting exposure time to CRISPR nuclease is the third primary strategy for reducing OTEs, which may have a concomitant reduction in on-target efficacy (171). Integrasedeficient lentiviral vector, ribonucleoprotein (RNP) complexes, and Cas 9 mRNA are all delivery systems generated for shortening Cas9 exposure (183-185). Despite the many advantages of using the RNP delivery system, immune adverse events must be noted (186). Other strategies to limit this exposure time are: (i) using doxycycline-inducible promoter controlling Cas9 expression in order to have a regulated expression; (ii) creating Cas9-intein, Cas9-degron, and split Cas to regulate Cas9 activity through cell-permeable compounds (Figure 4B); (iii) designing a selfrestriction construct consisting of a Cas9 that targets the system's gRNA; and (iv) using a Cas9 natural inhibitor (e.g., AcrIIA4) (187-193).

\section{DISCUSSION AND FUTURE PROSPECTS}

CRISPR/Cas9 has driven a paradigm shift in cancer treatment approaches since the day it was introduced. As mentioned above, despite sublime influences given by the CRISPR/Cas9 system in gene therapy, there are still many challenges that have to be considered.

The alternative Cas13 has been developed regarding the acknowledged challenges of Cas9-based CRISPR systems, including the risk of off-target toxicity, affecting wild type transcripts, and high molecular weight. Cas13 binds and cleaves single-stranded RNAs rather than DNA; therefore, it reduces the risk of off-target toxicity and wild type transcripts alterations. Moreover, the Cas13d subtype is known to maintain one of the lowest molecular weights among Cas enzymes. Thus, it can be introduced in target cells more easily through viral vectors. In conclusion, these RNA-targeting Cas enzymes would result in a considerable apprehension over transcriptome and RNA regulators functions in cancer cells (194). Besides, many types of other CRISPR related nucleases have been introduced, including Cpf1 and fnCas9 and nucleases that were made by changing amino acid residues in SpCas9 (SpCas9-HF1, eSpCas9, and HypaCas9) (174, 175). Application of these nucleases in cancer treatment and their probable advantages over more-studied SpCas9 should instead be evaluated in future studies.

Recent innovations in CRISPR-based systems have led to the emergence of new promising applications in cancer therapies. For instance, CDetection, as a CRISPR/Cas12b-based DNA detection system, has been developed to ease precise and sensitive DNA detection. Genetic variations, including Single Nucleotide Polymorphisms (SNPs), are also considered as cancer (especially sporadic cancers) etiologies $(195,196)$. Since the association of various SNPs with different cancers is well-established, precise DNA detection and its application in SNP genotyping would be favorable in the early clinical diagnosis of primary cancers. This precise DNA detection system is estimated to be able to detect over 20,000 known human disease-associated point mutations (197).

In summary, CRISPR and the above-mentioned advantages over other genome editing techniques could pave the road for cancer treatment in the future. This objective could be accomplished via using CRISPR as a potent instrument for gene therapy and identification of prognostic and predictive biomarkers, novel signaling pathways and targets, and new drugs in cancer treatment. CRISPR could also be used in identifying novel immune system-tumor interplays and augmenting cellular immunotherapies; however, related limitations and cautions should be noted before any interventions.

\section{AUTHOR CONTRIBUTIONS}

MA-K, MG, JKh, MB-S, and MJ performed the literature search and data analysis, drafted, and revised the work. MS and JKi critically revised the work. All authors contributed to the article and approved the submitted version. 


\section{REFERENCES}

1. Amer MH. Gene therapy for cancer: present status and future perspective. Mol Cell Therap. (2014) 2:27. doi: 10.1186/2052-8426-2-27

2. Chira S, Gulei D, Hajitou A, Berindan-Neagoe I. Restoring the p53 'Guardian' phenotype in p53-deficient tumor cells with CRISPR/Cas9. Trends Biotechnol. (2018) 36:653-60. doi: 10.1016/j.tibtech.2018.01.014

3. Maeder ML, Gersbach CA. Genome-editing technologies for gene and cell therapy. Mol Ther. (2016) 24:430-46. doi: 10.1038/mt.2016.10

4. Yin H, Xue W, Anderson DG. CRISPR-Cas: a tool for cancer research and therapeutics. Nat Rev Clin Oncol. (2019) 16:281-95. doi: 10.1038/s41571019-0166-8

5. Urnov FD, Rebar EJ, Holmes MC, Zhang HS, Gregory PD. Genome editing with engineered zinc finger nucleases. Nat Rev Genet. (2010) 11:636-46. doi: $10.1038 / \mathrm{nrg} 2842$

6. Carroll D. Genome engineering with zinc-finger nucleases. Genetics. (2011) 188:773-82. doi: 10.1534/genetics.111.131433

7. Cathomen T, Keith JJ. Zinc-finger nucleases: the next generation emerges. Mol Ther. (2008) 16:1200-7. doi: 10.1038/mt.2008.114

8. Cermak T, Doyle EL, Christian M, Wang L, Zhang Y, Schmidt C, et al. Efficient design and assembly of custom TALEN and other TAL effectorbased constructs for DNA targeting. Nucleic Acids Res. (2011) 39:e82. doi: $10.1093 / \mathrm{nar} / \mathrm{gkr} 218$

9. Miller JC, Tan S, Qiao G, Barlow KA, Wang J, Xia DF, et al. A TALE nuclease architecture for efficient genome editing. Nat Biotechnol. (2011) 29:143-8. doi: $10.1038 /$ nbt. 1755

10. Boch J. TALEs of genome targeting. Nat Biotechnol. (2011) 29:135-6. doi: 10.1038/nbt.1767

11. Cong L, Ran FA, Cox D, Lin S, Barretto R, Habib N, et al. Multiplex genome engineering using CRISPR/Cas systems. Science. (2013) 339:819-23. doi: 10. $1126 /$ science. 1231143

12. Mali P, Yang L, Esvelt KM, Aach J, Guell M, DiCarlo JE, et al. RNA-guided human genome engineering via Cas9. Science. (2013) 339:823-6. doi: 10. $1126 /$ science. 1232033

13. Damian M, Porteus MH. A crisper look at genome editing: RNA-guided genome modification. Mol Ther. (2013) 21:720-2. doi: 10.1038/mt.2013.46

14. Adli M. The CRISPR tool kit for genome editing and beyond. Nat Commun. (2018) 9:1911. doi: 10.1038/s41467-018-04252-2

15. Shalem O, Sanjana NE, Hartenian E, Shi X, Scott DA, Mikkelson T, et al. Genome-scale CRISPR-Cas9 knockout screening in human cells. Science. (2014) 343:84-7. doi: 10.1126/science.1247005

16. Qi LS, Larson MH, Gilbert LA, Doudna JA, Weissman JS, Arkin AP, et al. Repurposing CRISPR as an RNA-guided platform for sequence-specific control of gene expression. Cell. (2013) 152:1173-83. doi: 10.1016/j.cell.2013. 02.022

17. Behan FM, Iorio F, Picco G, Goncalves E, Beaver CM, Migliardi G, et al. Prioritization of cancer therapeutic targets using CRISPR-Cas9 screens. Nature. (2019) 568:511-6. doi: 10.1038/s41586-019-1103-9

18. Brouns SJ, Jore MM, Lundgren M, Westra ER, Slijkhuis RJ, Snijders AP, et al. Small CRISPR RNAs guide antiviral defense in prokaryotes. Science. (2008) 321:960-4. doi: 10.1126/science.1159689

19. Koonin EV, Krupovic M. Evolution of adaptive immunity from transposable elements combined with innate immune systems. Nat Rev Genet. (2015) 16:184-92. doi: 10.1038/nrg3859

20. Rath D, Amlinger L, Rath A, Lundgren M. The CRISPR-Cas immune system: biology, mechanisms and applications. Biochimie. (2015) 117:119-28. doi: 10.1016/j.biochi.2015.03.025

21. Wang H, La Russa M, Qi LS. CRISPR/Cas9 in Genome editing and beyond. Annu Rev Biochem. (2016) 85:227-64. doi: 10.1146/annurev-biochem060815-014607

22. Deltcheva E, Chylinski K, Sharma CM, Gonzales K, Chao Y, Pirzada ZA, et al. CRISPR RNA maturation by trans-encoded small RNA and host factor RNase III. Nature. (2011) 471:602-7. doi: 10.1038/nature09886

23. Jinek M, Chylinski K, Fonfara I, Hauer M, Doudna JA, Charpentier EA. programmable dual-RNA-guided DNA endonuclease in adaptive bacterial immunity. Science. (2012) 337:816-21. doi: 10.1126/science.1225829

24. Fonfara I, Richter H, Bratoviè M, Le Rhun A, Charpentier E. The CRISPR-associated DNA-cleaving enzyme Cpf1 also processes precursor CRISPR RNA. Nature. (2016) 532:517-21. doi: 10.1038/nature 17945

25. Lienert F, Lohmueller JJ, Garg A, Silver PA. Synthetic biology in mammalian cells: next generation research tools and therapeutics. Nat Rev Mol Cell Biol. (2014) 15:95-107. doi: 10.1038/nrm3738

26. Gaj T, Gersbach CA, Barbas CF III. ZFN, TALEN, and CRISPR/Cas-based methods for genome engineering. Trends Biotechnol. (2013) 31:397-405. doi: 10.1016/j.tibtech.2013.04.004

27. Nerys-Junior A, Braga-Dias LP, Pezzuto P, Cotta-de-Almeida V, Tanuri A. Comparison of the editing patterns and editing efficiencies of TALEN and CRISPR-Cas9 when targeting the human CCR5 gene. Genet Mol Biol. (2018) 41:167-79. doi: 10.1590/1678-4685-gmb-2017-0065

28. Salmon H, Remark R, Gnjatic S, Merad M. Host tissue determinants of tumour immunity. Nat Rev Cancer. (2019) 19:215-27. doi: 10.1038/s41568019-0125-9

29. Hanahan D, Weinberg RA. Hallmarks of cancer: the next generation. Cell. (2011) 144:646-74. doi: 10.1016/j.cell.2011.02.013

30. Albers J, Danzer C, Rechsteiner M, Lehmann H, Brandt LP, Hejhal $\mathrm{T}$, et al. A versatile modular vector system for rapid combinatorial mammalian genetics. J Clin Investigat. (2015) 125:1603-19. doi: 10.1172/JCI7 9743

31. Wang H, Sun W. CRISPR-mediated targeting of HER2 inhibits cell proliferation through a dominant negative mutation. Cancer Lett. (2017) 385:137-43. doi: 101016/jcanlet201610033

32. Tang H, Shrager JB. CRISPR/Cas-mediated genome editing to treat EGFRmutant lung cancer: a personalized molecular surgical therapy. EMBO Mol Med. (2016) 8:83-5. doi: 10.15252/emmm.201506006

33. Hayden A, Douglas J, Sommerlad M, Andrews L, Gould K, Hussain S, et al. The Nrf2 transcription factor contributes to resistance to cisplatin in bladder cancer. Urol Oncol. (2014) 32:806-14. doi: 10.1016/j.urolonc.2014. 02.006

34. Bialk P, Wang Y, Banas K, Kmiec EB. Functional gene knockout of NRF2 increases chemosensitivity of human lung cancer A549 Cells in vitro and in a xenograft mouse model. Mol Ther Oncolytics. (2018) 11:75-89. doi: 10.1016/j.omto.2018.10.002

35. Jones PA, Baylin SB. The epigenomics of cancer. Cell. (2007) 128:683-92. doi: 10.1016/j.cell.2007.01.029

36. Wang H, Guo R, Du Z, Bai L, Li L, Cui J, et al. Epigenetic targeting of granulin in hepatoma cells by synthetic CRISPR dCas9 epi-suppressors. Mol Ther Nucleic Acids. (2018) 11:23-33. doi: 10.1016/j.omtn.2018.01.002

37. Sato-Matsubara M, Kawada N. New player in tumor-stromal interaction: Granulin as a novel therapeutic target for pancreatic ductal adenocarcinoma liver metastasis. Hepatology. (2017) 65:374-6. doi: 10.1002/hep.28849

38. Yip CW, Cheung PF, Leung IC, Wong NC, Cheng CK, Fan ST, et al. Granulin-epithelin precursor interacts with heparan sulfate on liver cancer cells. Carcinogenesis. (2014) 35:2485-94. doi: 10.1093/carcin/bgul64

39. Hu Z, Yu L, Zhu D, Ding W, Wang X, Zhang C, et al. Disruption of HPV16-E7 by CRISPR/Cas system induces apoptosis and growth inhibition in HPV16 positive human cervical cancer cells. Biomed Res Int. (2014) 2014:612823. doi: 10.1155/2014/612823

40. Hsu DS, Kornepati AV, Glover W, Kennedy EM, Cullen BR. Targeting HPV16 DNA using CRISPR/Cas inhibits anal cancer growth in vivo. Future Virol (2018) 13:475-82. doi: 10.2217/fvl-2018-0010

41. Wang J, Quake SR. RNA-guided endonuclease provides a therapeutic strategy to cure latent herpesviridae infection. Proc Natl Acad Sci USA. (2014) 111:13157-62. doi: 10.1073/pnas.1410785111

42. Komor AC, Kim YB, Packer MS, Zuris JA, Liu DR. Programmable editing of a target base in genomic DNA without double-stranded DNA cleavage. Nature. (2016) 533:1476-4687.

43. Stephens PJ, Tarpey P, Davies H, Van Loo P, Greenman C, Wedge DC, et al. The landscape of cancer genes and mutational processes in breast cancer. Nature. (2012) 486:1476-4687.

44. Kuscu C, Parlak M, Tufan T, Yang J, Szlachta K, Wei X, et al. CRISPR-STOP: gene silencing through base-editing-induced nonsense mutations. Nature Methods. (2017) 14:1548-7105.

45. Matsuoka T, Yashiro M. Biomarkers of gastric cancer: current topics and future perspective. World J Gastroenterol. (2018) 24:2818-32. doi: 10.3748/ wjg.v24.i26.2818 
46. Zamborsky R, Kokavec M, Harsanyi S, Danisovic L. Identification of prognostic and predictive osteosarcoma biomarkers. Med Sci (Basel). (2019) 7:28. doi: 10.3390/medsci7020028

47. Tammi RH, Kultti A, Kosma VM, Pirinen R, Auvinen P, Tammi MI. Hyaluronan in human tumors: pathobiological and prognostic messages from cell-associated and stromal hyaluronan. Semin Cancer Biol. (2008) 18:288-95. doi: 10.1016/j.semcancer.2008.03.005

48. Yang GH, Fan J, Xu Y, Qiu SJ, Yang XR, Shi GM, et al. Osteopontin combined with CD44, a novel prognostic biomarker for patients with hepatocellular carcinoma undergoing curative resection. Oncologist. (2008) 13:1155-65. doi: 10.1634/theoncologist.2008-0081

49. Miletti-Gonzalez KE, Chen S, Muthukumaran N, Saglimbeni GN, Wu X, Yang J, et al. The CD44 receptor interacts with P-glycoprotein to promote cell migration and invasion in cancer. Cancer Res. (2005) 65:6660-7. doi: 10.1158/0008-5472.Can-04-3478

50. Choi $\mathrm{YH}, \mathrm{Yu}$ AM. ABC transporters in multidrug resistance and pharmacokinetics, and strategies for drug development. Curr Pharm Des. (2014) 20:793-807.

51. Mimeault M, Hauke R, Batra SK. Recent advances on the molecular mechanisms involved in the drug resistance of cancer cells and novel targeting therapies. Clin Pharmacol Ther. (2008) 83:673-91. doi: 10.1038/sj. clpt.6100296

52. Xiao Z, Wan J, Nur AA, Dou P, Mankin H, Liu T, et al. Targeting CD44 by CRISPR-Cas9 in multi-drug resistant osteosarcoma cells. Cell Physiol Biochem. (2018) 51:1879-93. doi: 10.1159/000495714

53. Radtke F, Clevers H. Self-renewal and cancer of the gut: two sides of a coin. Science. (2005) 307:1904-9. doi: 10.1126/science.1104815

54. Malliri A, Rygiel TP, van der Kammen RA, Song JY, Engers R, Hurlstone AF, et al. The rac activator Tiam1 is a Wnt-responsive gene that modifies intestinal tumor development. J Biol Chem. (2006) 281:543-8. doi: 10.1074/ jbc.M507582200

55. Izumi D, Toden S, Ureta E, Ishimoto T, Baba H, Goel A. TIAM1 promotes chemoresistance and tumor invasiveness in colorectal cancer. Cell Death Dis. (2019) 10:267. doi: 10.1038/s41419-019-1493-5

56. Vojta A, Dobrinic P, Tadic V, Bockor L, Korac P, Julg B, et al. Repurposing the CRISPR-Cas9 system for targeted DNA methylation. Nucleic Acids Res. (2016) 44:5615-28. doi: 10.1093/nar/gkw159

57. Qian P, De Kumar B, He XC, Nolte C, Gogol M, Ahn Y, et al. RetinoidSensitive epigenetic regulation of the hoxb cluster maintains normal hematopoiesis and inhibits leukemogenesis. Cell Stem Cell. (2018) 22:7404.e7. doi: 10.1016/j.stem.2018.04.012

58. Aquino-Jarquin G. Emerging role of CRISPR/Cas9 technology for MicroRNAs editing in cancer research. Cancer Res. (2017) 77:6812-7. doi: 10.1158/0008-5472.can-17-2142

59. Zhou SJ, Deng YL, Liang HF, Jaoude JC, Liu FY. Hepatitis B virus X protein promotes CREB-mediated activation of miR-3188 and Notch signaling in hepatocellular carcinoma. Cell Death Differ. (2017) 24:1577-87. doi: 10.1038/ cdd.2017.87

60. Feng Y, Sassi S, Shen JK, Yang X, Gao Y, Osaka E, et al. Targeting CDK11 in osteosarcoma cells using the CRISPR-Cas9 system. J Orthop Res. (2015) 33:199-207. doi: 10.1002/jor.22745

61. Mahmood N, Mihalcioiu C, Rabbani SA. Multifaceted role of the urokinasetype plasminogen activator (uPA) and its receptor (uPAR): diagnostic, prognostic, and therapeutic applications. Front Oncol. (2018) 8:24. doi: 10. 3389/fonc. 2018.00024

62. Smith HW, Marshall CJ. Regulation of cell signalling by uPAR. Nat Rev Mol Cell Biol. (2010) 11:23-36. doi: 10.1038/nrm2821

63. Wang K, Xing ZH, Jiang QW, Yang Y, Huang JR, Yuan ML, et al. Targeting uPAR by CRISPR/Cas9 system attenuates cancer malignancy and multidrug resistance. Front Oncol. (2019) 9:80. doi: 10.3389/fonc.2019.00080

64. Jahny E, Yang H, Liu B, Jahnke B, Lademann F, Knosel T, et al. The G proteincoupled receptor RAI3 Is an independent prognostic factor for pancreatic cancer survival and regulates proliferation via STAT3 phosphorylation. PLoS One. (2017) 12:e0170390. doi: 10.1371/journal.pone.0170390

65. Zhou H, Rigoutsos I. The emerging roles of GPRC5A in diseases. Oncoscience. (2014) 1:765-76. doi: 10.18632/oncoscience.104

66. Liu B, Yang H, Pilarsky C, Weber GF. The effect of GPRC5a on the proliferation, migration ability, chemotherapy resistance, and phosphorylation of GSK-3beta in pancreatic cancer. Int J Mol Sci. (2018) 19:1870. doi: 10.3390/ijms19071870

67. Porru M, Pompili L, Caruso C, Biroccio A, Leonetti C. Targeting KRAS in metastatic colorectal cancer: current strategies and emerging opportunities. $J$ Exp Clin Cancer Res. (2018) 37:57. doi: 10.1186/s13046-018-0719-1

68. Muzumdar MD, Chen PY, Dorans KJ, Chung KM, Bhutkar A, Hong E, et al. Survival of pancreatic cancer cells lacking KRAS function. Nat Commun. (2017) 8:1090. doi: 10.1038/s41467-017-00942-5

69. Beltran H, Rickman DS, Park K, Chae SS, Sboner A, MacDonald TY, et al. Molecular characterization of neuroendocrine prostate cancer and identification of new drug targets. Cancer Discov. (2011) 1:487-95. doi: 10. 1158/2159-8290.Cd-11-0130

70. Dardenne E, Beltran H, Benelli M, Gayvert K, Berger A, Puca L, et al. N-Myc induces an EZH2-mediated transcriptional program driving neuroendocrine prostate cancer. Cancer Cell. (2016) 30:563-77. doi: 10.1016/j.ccell.2016.09. 005

71. Mosquera JM, Beltran H, Park K, MacDonald TY, Robinson BD, Tagawa ST, et al. Concurrent AURKA and MYCN gene amplifications are harbingers of lethal treatment-related neuroendocrine prostate cancer. Neoplasia. (2013) 15:1-10.

72. Lee JK, Phillips JW, Smith BA, Park JW, Stoyanova T, McCaffrey EF, et al. NMyc drives neuroendocrine prostate cancer initiated from human prostate epithelial cells. Cancer Cell. (2016) 29:536-47. doi: 10.1016/j.ccell.2016.03. 001

73. Yin Y, Xu L, Chang Y, Zeng T, Chen X, Wang A, et al. N-Myc promotes therapeutic resistance development of neuroendocrine prostate cancer by differentially regulating miR-421/ATM pathway. Mol Cancer. (2019) 18:11. doi: 10.1186/s12943-019-0941-2

74. Roberts SA, Lawrence MS, Klimczak LJ, Grimm SA, Fargo D, Stojanov P, et al. An APOBEC cytidine deaminase mutagenesis pattern is widespread in human cancers. Nat Genet. (2013) 45:970-6. doi: 10.1038/ng.2702

75. Burns MB, Lackey L, Carpenter MA, Rathore A, Land AM, Leonard B, et al. APOBEC3B is an enzymatic source of mutation in breast cancer. Nature. (2013) 494:366-70. doi: 10.1038/nature11881

76. Burns MB, Temiz NA, Harris RS. Evidence for APOBEC3B mutagenesis in multiple human cancers. Nat Genet. (2013) 45:977-83. doi: 10.1038/ng.2701

77. Schmitt C, Lucius R, Synowitz M, Held-Feindt J, Hattermann K. APOBEC3B is expressed in human glioma, and influences cell proliferation and temozolomide resistance. Oncol Rep. (2018) 40:2742-9. doi: 10.3892/or.2018. 6698

78. Huo W, Zhao G, Yin J, Ouyang X, Wang Y, Yang C, et al. Lentiviral CRISPR/Cas9 vector mediated miR-21 gene editing inhibits the epithelial to mesenchymal transition in ovarian cancer cells. J Cancer. (2017) 8:57-64. doi: 10.7150/jca.16723

79. Yang D, Sun Y, Hu L, Zheng H, Ji P, Pecot CV, et al. Integrated analyses identify a master microRNA regulatory network for the mesenchymal subtype in serous ovarian cancer. Cancer Cell. (2013) 23:186-99. doi: 10.1016/ j.ccr.2012.12.020

80. Koike-Yusa H, Li Y, Tan EP, Velasco-Herrera Mdel C, Yusa K. Genome-wide recessive genetic screening in mammalian cells with a lentiviral CRISPRguide RNA library. Nat Biotechnol. (2014) 32:267-73. doi: 10.1038/nbt.2800

81. Zhou Y, Zhu S, Cai C, Yuan P, Li C, Huang Y, et al. High-throughput screening of a CRISPR/Cas9 library for functional genomics in human cells. Nature. (2014) 509:487-91. doi: 10.1038/nature13166

82. Zhou X, Li R, Jing R, Zuo B, Zheng Q. Genome-wide CRISPR knockout screens identify ADAMTSL3 and PTEN genes as suppressors of HCC proliferation and metastasis, respectively. J Cancer Res Clin Oncol. (2020) 146:1509-21. doi: 10.1007/s00432-020-03207-9

83. Du X, Shen X, Dai L, Bi F, Zhang H, Lu C. PSMD12 promotes breast cancer growth via inhibiting the expression of pro-apoptotic genes. Biochem Biophys Res Commun. (2020) 526:368-74. doi: 10.1016/j.bbrc.2020. 03.095

84. Yang B, Kuang J, Wu C, Zhou W, Zhu S, Jiang H, et al. Screening Genes promoting exit from naive pluripotency based on genome-scale CRISPRCas9 knockout. Stem Cells Int. (2020) 2020:8483035. doi: 10.1155/2020/ 8483035

85. Hu M, Fu X, Si Z, Li C, Sun J, Du X, et al. Identification of differently expressed genes associated with prognosis and growth in colon 
adenocarcinoma based on integrated bioinformatics analysis. Front Genet. (2019) 10:1245. doi: 10.3389/fgene.2019.01245

86. Wheeler LJ, Watson ZL, Qamar L, Yamamoto TM, Sawyer BT, Sullivan KD, et al. Multi-omic approaches identify metabolic and autophagy regulators important in ovarian cancer dissemination. iScience. (2019) 19:474-91. doi: 10.1016/j.isci.2019.07.049

87. Ouyang Q, Liu Y, Tan J, Li J, Yang D, Zeng F, et al. Loss of ZNF587B and SULF1 contributed to cisplatin resistance in ovarian cancer cell lines based on Genome-scale CRISPR/Cas9 screening. Am J Cancer Res. (2019) 9:988-98.

88. Sun W, He B, Yang B, Hu W, Cheng S, Xiao H, et al. Genome-wide CRISPR screen reveals SGOL1 as a druggable target of sorafenib-treated hepatocellular carcinoma. Lab Invest. (2018) 98:734-44. doi: 10.1038/s41374018-0027-6

89. Wang T, Wei JJ, Sabatini DM, Lander ES. Genetic screens in human cells using the CRISPR-Cas9 system. Science. (2014) 343:80-4. doi: 10.1126/ science. 1246981

90. Manguso RT, Pope HW, Zimmer MD, Brown FD, Yates KB, Miller BC, et al. In vivo CRISPR screening identifies Ptpn2 as a cancer immunotherapy target. Nature. (2017) 547:413-8. doi: 10.1038/nature23270

91. Zhang JP, Song Z, Wang HB, Lang L, Yang YZ, Xiao W, et al. A novel model of controlling PD-L1 expression in ALK(+) anaplastic large cell lymphoma revealed by CRISPR screening. Blood. (2019) 134:171-85. doi: 10.1182/blood. 2019001043

92. Shi CX, Kortüm KM, Zhu YX, Bruins LA, Jedlowski P, Votruba PG, et al. CRISPR genome-wide screening identifies dependence on the proteasome subunit PSMC6 for bortezomib sensitivity in multiple myeloma. Mol Cancer Ther. (2017) 16:2862-70. doi: 10.1158/1535-7163.Mct-17-0130

93. Liu J, Song T, Zhou W, Xing L, Wang S, Ho M, et al. A genome-scale CRISPRCas9 screening in myeloma cells identifies regulators of immunomodulatory drug sensitivity. Leukemia. (2019) 33:171-80. doi: 10.1038/s41375-018$0205-\mathrm{y}$

94. Tzelepis K, Koike-Yusa H, De Braekeleer E, Li Y, Metzakopian E, Dovey OM, et al. A CRISPR dropout screen identifies genetic vulnerabilities and therapeutic targets in acute myeloid leukemia. Cell Rep. (2016) 17:1193-205. doi: 10.1016/j.celrep.2016.09.079

95. Yamauchi T, Masuda T, Canver MC, Seiler M, Semba Y, Shboul M, et al. Genome-wide CRISPR-Cas9 screen identifies leukemia-specific dependence on a Pre-mRNA metabolic pathway regulated by DCPS. Cancer Cell. (2018) 33:386-400.e5. doi: 10.1016/j.ccell.2018.01.012

96. Echeverri CJ, Beachy PA, Baum B, Boutros M, Buchholz F, Chanda SK, et al. Minimizing the risk of reporting false positives in large-scale RNAi screens. Nat Methods. (2006) 3:777-9. doi: 10.1038/nmeth1006-777

97. Munoz DM, Cassiani PJ, Li L, Billy E, Korn JM, Jones MD, et al. CRISPR screens provide a comprehensive assessment of cancer vulnerabilities but generate false-positive hits for highly amplified genomic regions. Cancer Discov. (2016) 6:900-13. doi: 10.1158/2159-8290.Cd-16-0178

98. Shimizu K, Iyoda T, Okada M, Yamasaki S, Fujii SI. Immune suppression and reversal of the suppressive tumor microenvironment. Int Immunol. (2018) 30:445-54. doi: 10.1093/intimm/dxy042

99. Buoncervello M, Gabriele L, Toschi E. The janus face of tumor microenvironment targeted by immunotherapy. Int J Mol Sci. (2019) 20:4320. doi: 10.3390/ijms20174320

100. Thommen DS, Schumacher TN. T cell dysfunction in cancer. Cancer Cell. (2018) 33:547-62. doi: 10.1016/j.ccell.2018.03.012

101. Chung W, Eum HH, Lee HO, Lee KM, Lee HB, Kim KT, et al. Singlecell RNA-seq enables comprehensive tumour and immune cell profiling in primary breast cancer. Nat Commun. (2017) 8:15081. doi: 10.1038/ ncomms 15081

102. Maus MV, Fraietta JA, Levine BL, Kalos M, Zhao Y, June CH. Adoptive immunotherapy for cancer or viruses. Annu Rev Immunol. (2014) 32:189225. doi: 10.1146/annurev-immunol-032713-120136

103. Rosenberg SA, Restifo NP, Yang JC, Morgan RA, Dudley ME. Adoptive cell transfer: a clinical path to effective cancer immunotherapy. Nat Rev Cancer. (2008) 8:299-308. doi: 10.1038/nrc2355

104. Mantovani A, Sica A, Sozzani S, Allavena P, Vecchi A, Locati M. The chemokine system in diverse forms of macrophage activation and polarization. Trends Immunol. (2004) 25:677-86. doi: 10.1016/j.it.2004. 09.015
105. Mills CD. M1 and M2 macrophages: oracles of health and disease. Crit Rev Immunol. (2012) 32:463-88. doi: 10.1615/critrevimmunol.v32.i6.10

106. Martinez FO, Gordon S. The M1 and M2 paradigm of macrophage activation: time for reassessment. F1000Prime Rep. (2014) 6:13. doi: 10.12703/p6-13

107. Mills CD, Kincaid K, Alt JM, Heilman MJ, Hill AMM-. 1/M-2 macrophages and the Th1/Th2 paradigm. J Immunol. (2000) 164:6166-73. doi: 10.4049/ jimmunol.164.12.6166

108. Ray M, Lee YW, Hardie J, Mout R, Yesilbag Tonga G, Farkas ME, et al. CRISPRed macrophages for cell-based cancer immunotherapy. Bioconjug Chem. (2018) 29:445-50. doi: 10.1021/acs.bioconjchem.7b00768

109. Sossey-Alaoui K, Pluskota E, Bialkowska K, Szpak D, Parker Y, Morrison CD, et al. Kindlin-2 regulates the growth of breast cancer tumors by activating CSF-1-mediated macrophage infiltration. Cancer Res. (2017) 77:5129-41. doi: 10.1158/0008-5472.Can-16-2337

110. Wei J, Marisetty A, Schrand B, Gabrusiewicz K, Hashimoto Y, Ott M, et al. Osteopontin mediates glioblastoma-associated macrophage infiltration and is a potential therapeutic target. J Clin Investigat. (2019) 129:137-49. doi: $10.1172 /$ jci121266

111. Wang R, Liu Y, Liu L, Chen M, Wang X, Yang J, et al. Tumor cells induce LAMP2a expression in tumor-associated macrophage for cancer progression. EBioMedicine. (2019) 40:118-34. doi: 10.1016/j.ebiom.2019.01.045

112. Jin K, Pandey NB, Popel AS. Crosstalk between stromal components and tumor cells of TNBC via secreted factors enhances tumor growth and metastasis. Oncotarget. (2017) 8:60210-22. doi: 10.18632/oncotarget.19417

113. Ubil E, Caskey L, Holtzhausen A, Hunter D, Story C, Earp HS. Tumorsecreted Pros1 inhibits macrophage M1 polarization to reduce antitumor immune response. J Clin Investigat. (2018) 128:2356-69. doi: 10.1172/ jci97354

114. Mason C, Brindley DA, Culme-Seymour EJ, Davie NL. Cell therapy industry: billion dollar global business with unlimited potential. Regen Med. (2011) 6:265-72. doi: 10.2217/rme.11.28

115. Essand M, Loskog AS. Genetically engineered T cells for the treatment of cancer. J Intern Med. (2013) 273:166-81. doi: 10.1111/joim.12020

116. D’Aloia MM, Zizzari IG, Sacchetti B, Pierelli L, Alimandi M. CAR-T cells: the long and winding road to solid tumors. Cell Death Dis. (2018) 9:282.

117. Depil S, Duchateau P, Grupp SA, Mufti G, Poirot L. 'Off-the-shelf' allogeneic CAR T cells: development and challenges. Nature Reviews Drug Discovery. (2020) 19:185-99. doi: 10.1038/s41573-019-0051-2

118. Köhl U, Arsenieva S, Holzinger A, Abken H. CAR T cells in trials: recent achievements and challenges that remain in the production of modified $\mathrm{T}$ Cells for clinical applications. Hum Gene Ther. (2018) 29:559-68. doi: 10. 1089/hum.2017.254

119. Zhang L, Chu J, Yu J, Wei W. Cellular and molecular mechanisms in graft-versus-host disease. J Leukoc Biol. (2016) 99:279-87. doi: 10.1189/jlb. 4RU0615-254RR

120. Marino J, Paster J, Benichou G. Allorecognition by T lymphocytes and allograft rejection. Front Immunol. (2016) 7:582. doi: 10.3389/fimmu.2016. 00582

121. Poirot L, Philip B, Schiffer-Mannioui C, Le Clerre D, Chion-Sotinel I, Derniame S, et al. Multiplex genome-edited T-cell manufacturing platform for "Off-the-Shelf" adoptive T-cell immunotherapies. Cancer Res. (2015) 75:3853-64. doi: 10.1158/0008-5472.Can-14-3321

122. Qasim W, Zhan H, Samarasinghe S, Adams S, Amrolia P, Stafford S, et al. Molecular remission of infant B-ALL after infusion of universal TALEN geneedited CAR T cells. Sci Transl Med. (2017) 9:374. doi: 10.1126/scitranslmed. aaj2013

123. Zhao J, Lin Q, Song Y, Liu D. Universal CARs, universal T cells, and universal CAR T cells. J Hematol Oncol. (2018) 11:132. doi: 10.1186/s13045-0180677-2

124. Eyquem J, Mansilla-Soto J, Giavridis T, van der Stegen SJ, Hamieh $\mathrm{M}$, Cunanan $\mathrm{KM}$, et al. Targeting a CAR to the TRAC locus with CRISPR/Cas9 enhances tumour rejection. Nature. (2017) 543:113-7. doi: 10.1038 /nature21405

125. Ren J, Liu X, Fang C, Jiang S, June CH, Zhao Y. Multiplex genome editing to generate universal CAR T cells resistant to PD1 inhibition. Clin Cancer Res. (2017) 23:2255-66. doi: 10.1158/1078-0432.ccr-16-1300

126. Choi BD, Yu X, Castano AP, Darr H, Henderson DB, Bouffard AA, et al. CRISPR-Cas9 disruption of PD-1 enhances activity of universal EGFRvIII 
CAR T cells in a preclinical model of human glioblastoma. J Immunother Cancer. (2019) 7:304. doi: 10.1186/s40425-019-0806-7

127. van Loenen MM, de Boer R, Amir AL, Hagedoorn RS, Volbeda GL, Willemze R, et al. Mixed T cell receptor dimers harbor potentially harmful neoreactivity. Proc Natl Acad Sci USA. (2010) 107:10972-7. doi: 10.1073/ pnas. 1005802107

128. Heemskerk MH, Hagedoorn RS, van der Hoorn MA, van der Veken LT, Hoogeboom M, Kester MG, et al. Efficiency of T-cell receptor expression in dual-specific $\mathrm{T}$ cells is controlled by the intrinsic qualities of the TCR chains within the TCR-CD3 complex. Blood. (2007) 109:235-43. doi: 10. 1182/blood-2006-03-013318

129. Provasi E, Genovese P, Lombardo A, Magnani Z, Liu PQ, Reik A, et al. Editing $T$ cell specificity towards leukemia by zinc finger nucleases and lentiviral gene transfer. Nat Med. (2012) 18:807-15. doi: 10.1038/nm.2700

130. Stadtmauer EA, Fraietta JA, Davis MM, Cohen AD, Weber KL, Lancaster E, et al. CRISPR-engineered T cells in patients with refractory cancer. Science. (2020) 367:7365. doi: 10.1126/science.aba7365

131. John LB, Devaud C, Duong CPM, Yong CS, Beavis PA, Haynes NM, et al. Anti-PD- 1 antibody therapy potently enhances the eradication of established tumors by gene-modified T cells. Clin Cancer Res. (2013) 19:5636-46. doi: 10.1158/078-0432.CCR-13-58

132. Topalian SL, Drake CG, Pardoll DM. Immune checkpoint blockade: a common denominator approach to cancer therapy. Cancer Cell. (2015) 27:450-61. doi: 10.1016/j.ccell.2015.03.001

133. Su S, Hu B, Shao J, Shen B, Du J, Du Y, et al. CRISPR-Cas9 mediated efficient PD-1 disruption on human primary T cells from cancer patients. Sci Rep. (2016) 6:20070. doi: 10.1038/srep20070

134. Hu B, Zou Y, Zhang L, Tang J, Niedermann G, Firat E, et al. Nucleofection with plasmid DNA for CRISPR/Cas9-mediated inactivation of programmed cell death protein 1 in CD133-specific CAR T cells. Hum Gene Ther. (2018) 30:446-58. doi: 10.1089/hum.2017.234

135. Dai X, Park JJ, Du Y, Kim HR, Wang G, Errami Y, et al. One-step generation of modular CAR-T cells with AAV-Cpf1. Nat Methods. (2019) 16:247-54. doi: 10.1038/s41592-019-0329-7

136. Rupp LJ, Schumann K, Roybal KT, Gate RE, Ye CJ, Lim WA, et al. CRISPR/Cas9-mediated PD-1 disruption enhances anti-tumor efficacy of human chimeric antigen receptor T cells. (A.M., K.S.). A.M. serves as an advisor to Juno Therapeutics and PACT Therapeutics and the Marson lab has received sponsored research support from Juno Therapeutics and Epinomics. L.J.R. is an employee of Cell Design Labs, K.T.R. is an advisor to Cell Design Labs, and W.A.L. is a founder of Cell Design Labs and a member of its scientific advisory board. A.M. is an advisor to Juno Therapeutics. Sci Rep. (2017) 7:737. doi: 10.1038/s41598-017-00462-8

137. Guo X, Jiang H, Shi B, Zhou M, Zhang H, Shi Z, et al. Disruption of PD1 enhanced the anti-tumor activity of chimeric antigen receptor $\mathrm{T}$ cells against hepatocellular carcinoma. Front Pharmacol. (2018) 9:1118. doi: 10. 3389/fphar.2018.01118

138. Nakazawa T, Natsume A, Nishimura F, Morimoto T, Matsuda R, Nakamura $\mathrm{M}$, et al. Effect of CRISPR/Cas9-Mediated PD-1-disrupted primary human third-generation CAR-T cells targeting EGFRvIII on In Vitro human glioblastoma cell growth. Cells. (2020) 9:998. doi: 10.3390/cells9040998

139. Cyranoski D. CRISPR gene-editing tested in a person for the first time. Nature. (2016) 539:479. doi: 10.1038/nature.2016.20988

140. Zhang Y, Zhang X, Cheng C, Mu W, Liu X, Li N, et al. CRISPR-Cas9 mediated LAG-3 disruption in CAR-T cells. Front Med. (2017) 11:554-62. doi: 10.1007/s11684-017-0543-6

141. Kunkele A, Johnson AJ, Rolczynski LS, Chang CA, Hoglund V, Kelly-Spratt KS, et al. Functional tuning of CARs Reveals signaling threshold above which CD8+ CTL antitumor potency is attenuated due to cell Fas-FasL-dependent AICD. Cancer Immunol Res. (2015) 3:368-79. doi: 10.1158/2326-6066.cir14-0200

142. Singh N, Shi J, June CH, Ruella M. Genome-editing technologies in adoptive T cell immunotherapy for cancer. Curr Hematol Malig Rep. (2017) 12:522-9. doi: 10.1007/s11899-017-0417-7

143. Sterner RM, Sakemura R, Cox MJ, Yang N, Khadka RH, Forsman $\mathrm{CL}$, et al. GM-CSF inhibition reduces cytokine release syndrome and neuroinflammation but enhances CAR-T cell function in xenografts. Blood. (2019) 133:697-709. doi: 10.1182/blood-2018-10-881722
144. Gomes-Silva D, Srinivasan M, Sharma S, Lee CM, Wagner DL, Davis TH, et al. CD7-edited T cells expressing a CD7-specific CAR for the therapy of T-cell malignancies. Blood. (2017) 130:285-96. doi: 10.1182/blood-2017-01761320

145. Cooper ML, Choi J, Staser K, Ritchey JK, Devenport JM, Eckardt K, et al. An "off-the-shelf" fratricide-resistant CAR-T for the treatment of T cell hematologic malignancies. Leukemia. (2018) 32:1970-83. doi: 10.1038/ s41375-018-0065-5

146. Jung IY, Kim YY, Yu HS, Lee M, Kim S, Lee J. CRISPR/Cas9-mediated knockout of DGK improves antitumor activities of human T cells. Cancer Res. (2018) 78:4692-703. doi: 10.1158/0008-5472.can-18-0030

147. Fraietta JA, Nobles CL, Sammons MA, Lundh S, Carty SA, Reich TJ, et al. Disruption of TET2 promotes the therapeutic efficacy of CD19-targeted T cells. Nature. (2018) 558:307-12. doi: 10.1038/s41586-018-0178-z

148. Kelly E, Russell SJ. History of oncolytic viruses: genesis to genetic engineering. Mol Ther. (2007) 15:651-9. doi: 10.1038/sj.mt.6300108

149. Russell SJ, Peng KW, Bell JC. Oncolytic virotherapy. Nat Biotechnol. (2012) 30:658-70. doi: 10.1038/nbt.2287

150. Raja J, Ludwig JM, Gettinger SN, Schalper KA, Kim HS. Oncolytic virus immunotherapy: future prospects for oncology. J Immunother Cancer. (2018) 6:140. doi: 10.1186/s40425-018-0458-z

151. Kohlhapp FJ, Kaufman HL. Molecular pathways: mechanism of action for talimogene laherparepvec, a new oncolytic virus immunotherapy. Clin Cancer Res. (2016) 22:1048-54. doi: 10.1158/1078-0432.Ccr-15-2667

152. Jhawar SR, Thandoni A, Bommareddy PK, Hassan S, Kohlhapp FJ, Goyal S, et al. Oncolytic viruses-natural and genetically engineered cancer immunotherapies. Front Oncol. (2017) 7:202. doi: 10.3389/fonc.2017.00202

153. Chiocca EA, Rabkin SD. Oncolytic viruses and their application to cancer immunotherapy. Cancer Immunol Res. (2014) 2:295-300. doi: 10.1158/23266066.Cir-14-0015

154. Lawler SE, Speranza MC, Cho CF, Chiocca EA. Oncolytic viruses in cancer treatment: a review. JAMA Oncol. (2017) 3:841-9. doi: 10.1001/jamaoncol. 2016.2064

155. Fountzilas C, Patel S, Mahalingam D. Review: oncolytic virotherapy, updates and future directions. Oncotarget. (2017) 8:102617-39. doi: 10.18632/ oncotarget. 18309

156. Bi Y, Sun L, Gao D, Ding C, Li Z, Li Y, et al. High-efficiency targeted editing of large viral genomes by RNA-guided nucleases. PLoS Pathog. (2014) 10:e1004090. doi: 10.1371/journal.ppat.1004090

157. Lin C, Li H, Hao M, Xiong D, Luo Y, Huang C, et al. Increasing the efficiency of CRISPR/Cas9-mediated precise genome editing of HSV-1 virus in human cells. Sci Rep. (2016) 6:34531. doi: 10.1038/srep34531

158. Suenaga T, Kohyama M, Hirayasu K, Arase H. Engineering large viral DNA genomes using the CRISPR-Cas9 system. Microbiol Immunol. (2014) 58:513-22. doi: 10.1111/1348-0421.12180

159. Guo ZS, Lu B, Guo Z, Giehl E, Feist M, Dai E, et al. Vaccinia virus-mediated cancer immunotherapy: cancer vaccines and oncolytics. Not applicable. COMPETING INTERESTS: ZSG and DLB serve as scientific advisors to ICell Kealex Therapeutics. DLB has financial interest with SillaJen Biotherapeutics. Pexa-Vec, a product discussed in this review, is manufactured by SillaJen. All other authors declare no conflict of interest. PUBLISHER'S NOTE: Springer Nature remains neutral with regard to jurisdictional claims in published maps and institutional affiliations. J Immunother Cancer. (2019) 7:6. doi: 10.1186/s40425-018-0495-7

160. Yuan M, Zhang W, Wang J, Al Yaghchi C, Ahmed J, Chard L, et al. Efficiently editing the vaccinia virus genome by using the CRISPR-Cas9 system. $J$ Virol. (2015) 89:5176-9. doi: 10.1128/jvi.00339-15

161. Cai L, Hu H, Duan H, Li Y, Zou Z, Luo K, et al. The construction of a new oncolytic herpes simplex virus expressing murine interleukin-15 with gene-editing technology. J Med Virol. (2020) 20:25691. doi: 10.1002/jmv. 25691

162. Phelps MP, Yang H, Patel S, Rahman MM, McFadden G, Chen E. Oncolytic virus-mediated RAS targeting in rhabdomyosarcoma. Mol Ther Oncolytics. (2018) 11:52-61. doi: 10.1016/j.omto.2018.09.001

163. Nachmanson D, Lian S, Schmidt EK, Hipp MJ, Baker KT, Zhang Y, et al. CRISPR-DS: an efficient, low DNA input method for ultraaccurate sequencing. bioRxiv. (2017). [Preprint]. doi: 10.1101/20 7027 
164. Charlesworth CT, Deshpande PS, Dever DP, Camarena J, Lemgart VT, Cromer MK, et al. Identification of preexisting adaptive immunity to Cas9 proteins in humans. Nat Med. (2019) 25:249-54. doi: 10.1038/s41591-0180326-x

165. Haapaniemi E, Botla S, Persson J, Schmierer B, Taipale J. CRISPR-Cas9 genome editing induces a p53-mediated DNA damage response. Nat Med. (2018) 24:927-30. doi: 10.1038/s41591-018-0049-z

166. Ihry RJ, Worringer KA, Salick MR, Frias E, Ho D, Theriault K, et al. p53 inhibits CRISPR-Cas9 engineering in human pluripotent stem cells. Nat Med. (2018) 24:939-46. doi: 10.1038/s41591-018-0050-6

167. Kosicki M, Tomberg K, Bradley A. Repair of double-strand breaks induced by CRISPR-Cas9 leads to large deletions and complex rearrangements. Nat Biotechnol. (2018) 36:765-71. doi: 10.1038/nbt.4192

168. Straathof KC, Pule MA, Yotnda P, Dotti G, Vanin EF, Brenner MK, et al. An inducible caspase 9 safety switch for T-cell therapy. Blood. (2005) 105:424754. doi: 10.1182/blood-2004-11-4564

169. Bonifant CL, Jackson HJ, Brentjens RJ, Curran KJ. Toxicity and management in CAR T-cell therapy. Mol Ther Oncolytics. (2016) 3:16011. doi: 10.1038/ mto.2016.11

170. Zhang XH, Tee LY, Wang XG, Huang QS, Yang SH. Off-target Effects in CRISPR/Cas9-mediated genome engineering. Mol Ther Nucleic Acids. (2015) 4:e264. doi: 10.1038/mtna.2015.37

171. Kimberland ML, Hou W, Alfonso-Pecchio A, Wilson S, Rao Y, Zhang S, et al. Strategies for controlling CRISPR/Cas9 off-target effects and biological variations in mammalian genome editing experiments. J Biotechnol. (2018) 284:91-101. doi: 10.1016/j.jbiotec.2018.08.007

172. Cromwell CR, Sung K, Park J, Krysler AR, Jovel J, Kim SK, et al. Incorporation of bridged nucleic acids into CRISPR RNAs improves Cas 9 endonuclease specificity. Nat Commun. (2018) 9:1448. doi: 10.1038/s41467-018-03927-0

173. Fu Y, Sander JD, Reyon D, Cascio VM, Joung JK. Improving CRISPRCas nuclease specificity using truncated guide RNAs. Nat Biotechnol. (2014) 32:279-84. doi: 10.1038/nbt.2808

174. Chen F, Ding X, Feng Y, Seebeck T, Jiang Y, Davis GD. Targeted activation of diverse CRISPR-Cas systems for mammalian genome editing via proximal CRISPR targeting. Nat Commun. (2017) 8:14958. doi: 10.1038/ ncomms 14958

175. Kleinstiver BP, Tsai SQ, Prew MS, Nguyen NT, Welch MM, Lopez JM, et al. Genome-wide specificities of CRISPR-Cas Cpf1 nucleases in human cells. Nat Biotechnol. (2016) 34:869-74. doi: 10.1038/nbt.3620

176. Chen JS, Dagdas YS, Kleinstiver BP, Welch MM, Sousa AA, Harrington LB, et al. Enhanced proofreading governs CRISPR-Cas9 targeting accuracy. Nature. (2017) 550:407-10. doi: 10.1038/nature24268

177. Kleinstiver BP, Pattanayak V, Prew MS, Tsai SQ, Nguyen NT, Zheng Z, et al. High-fidelity CRISPR-Cas9 nucleases with no detectable genome-wide off-target effects. Nature. (2016) 529:490-5. doi: 10.1038/nature16526

178. Slaymaker IM, Gao L, Zetsche B, Scott DA, Yan WX, Zhang F. Rationally engineered Cas9 nucleases with improved specificity. Science. (2016) 351:848. doi: $10.1126 /$ science.aad5227

179. Hu JH, Miller SM, Geurts MH, Tang W, Chen L, Sun N, et al. Evolved Cas9 variants with broad PAM compatibility and high DNA specificity. Nature. (2018) 556:57-63. doi: 10.1038/nature26155

180. Kleinstiver BP, Prew MS, Tsai SQ, Nguyen NT, Topkar VV, Zheng Z, et al. Broadening the targeting range of Staphylococcus aureus CRISPR-Cas9 by modifying PAM recognition. Nat Biotechnol. (2015) 33:1293-8. doi: 10.1038/ nbt.3404

181. Guilinger JP, Thompson DB, Liu DR. Fusion of catalytically inactive Cas9 to FokI nuclease improves the specificity of genome modification. Nat Biotechnol. (2014) 32:577-82. doi: 10.1038/nbt.2909

182. Ran FA, Hsu PD, Lin CY, Gootenberg JS, Konermann S, Trevino AE, et al. Double nicking by RNA-guided CRISPR Cas9 for enhanced genome editing specificity. Cell. (2013) 154:1380-9. doi: 10.1016/j.cell.2013.08.021

183. Kim S, Kim D, Cho SW, Kim J, Kim JS. Highly efficient RNA-guided genome editing in human cells via delivery of purified Cas9 ribonucleoproteins. Genome Res. (2014) 24:1012-9. doi: 10.1101/gr.171322.113

184. Liang X, Potter J, Kumar S, Zou Y, Quintanilla R, Sridharan M, et al. Rapid and highly efficient mammalian cell engineering via Cas9 protein transfection. J Biotechnol. (2015) 208:44-53. doi: 10.1016/j.jbiotec.2015. 04.024
185. Ortinski PI, O’Donovan B, Dong X, Kantor B. Integrase-deficient lentiviral vector as an all-in-one platform for highly efficient CRISPR/Cas9-mediated gene editing. Mol Ther Methods Clin Dev. (2017) 5:153-64. doi: 10.1016/j. omtm.2017.04.002

186. Kim S, Koo T, Jee HG, Cho HY, Lee G, Lim DG, et al. CRISPR RNAs trigger innate immune responses in human cells. Genome Res. (2018) 117:231936. doi: $10.1101 / \mathrm{gr}$

187. Cao J, Wu L, Zhang SM, Lu M, Cheung WK, Cai W, et al. An easy and efficient inducible CRISPR/Cas9 platform with improved specificity for multiple gene targeting. Nucleic Acids Res. (2016) 44:e149. doi: 10.1093/nar/ gkw660

188. Chen Y, Liu X, Zhang Y, Wang H, Ying H, Liu M, et al. A self-restricted CRISPR system to reduce off-target effects. Mol Ther. (2016) 24:1508-10. doi: $10.1038 / \mathrm{mt} .2016 .172$

189. Davis KM, Pattanayak V, Thompson DB, Zuris JA, Liu DR. Small moleculetriggered Cas9 protein with improved genome-editing specificity. Nat Chem Biol. (2015) 11:316-8. doi: 10.1038/nchembio.1793

190. Maji B, Moore CL, Zetsche B, Volz SE, Zhang F, Shoulders MD, et al. Multidimensional chemical control of CRISPR-Cas9. Nat Chem Biol. (2017) 13:9-11. doi: 10.1038/nchembio.2224

191. Rauch BJ, Silvis MR, Hultquist JF, Waters CS, McGregor MJ, Krogan NJ, et al. Inhibition of CRISPR-Cas9 with bacteriophage proteins. Cell. (2017) 168:150-8.e10. doi: 10.1016/j.cell.2016.12.009

192. Senturk S, Shirole NH, Nowak DG, Corbo V, Pal D, Vaughan A, et al. Rapid and tunable method to temporally control gene editing based on conditional Cas9 stabilization. Nat Commun. (2017) 8:14370. doi: 10.1038/ ncomms 14370

193. Zhu Z, Gonzalez F, Huangfu D. The iCRISPR platform for rapid genome editing in human pluripotent stem cells. Methods Enzymol. (2014) 546:21550. doi: 10.1016/b978-0-12-801185-0.00011-8

194. Granados-Riveron JT, Aquino-Jarquin G. CRISPR-Cas13 precision transcriptome engineering in cancer. Cancer Res. (2018) 78:4107-13.

195. Abd El-Fattah AA, Sadik NAH, Shaker OA-O, Mohamed Kamal AA-O. Single nucleotide polymorphism in SMAD7 and CHI3L1 and colorectal cancer risk. Mediators Inflamm. (2018) 2018:9853192.

196. Philpott C, Tovell H, Frayling IM, Cooper DN, Upadhyaya M. The NF1 somatic mutational landscape in sporadic human cancers. Hum Genomics. (2017) 11:1479-7364

197. Teng F, Guo L, Cui T, Wang XG, Xu K, Gao Q, et al. CDetection: CRISPRCas12b-based DNA detection with sub-attomolar sensitivity and single-base specificity. Genome Biol. (2019) 20:132.

198. Anders C, Niewoehner O, Duerst A, Jinek M. Structural basis of PAMdependent target DNA recognition by the Cas 9 endonuclease. Nature. (2014) 513:569-73. doi: 10.1038/nature13579

199. Miyaoka Y, Berman JR, Cooper SB, Mayerl SJ, Chan AH, Zhang B, et al. Systematic quantification of HDR and NHEJ reveals effects of locus, nuclease, and cell type on genome-editing. Sci Rep. (2016) 6:23549. doi: 10.1038/ srep23549

200. Wang B, Li K, Wang A, Reiser M, Saunders T, Lockey RF, et al. Highly efficient CRISPR/HDR-mediated knock-in for mouse embryonic stem cells and zygotes. BioTechniques. (2015) 59:201-2. doi: 10.2144/00011 4339

201. Parsi KM, Hennessy E, Kearns N, Maehr R. Using an inducible CRISPRdCas9-KRAB effector system to dissect transcriptional regulation in human embryonic stem cells. Methods Mol Biol (Clifton N J). (2017) 1507:221-33. doi: 10.1007/978-1-4939-6518-2_16

202. Maeder ML, Linder SJ, Cascio VM, Fu Y, Ho QH, Joung JK. CRISPR RNA-guided activation of endogenous human genes. Nat Methods. (2013) 10:977-9. doi: 10.1038/nmeth.2598

203. Perez-Pinera P, Kocak DD, Vockley CM, Adler AF, Kabadi AM, Polstein LR, et al. RNA-guided gene activation by CRISPR-Cas9-based transcription factors. Nat Methods. (2013) 10:973-6. doi: 10.1038/nmeth.2600

204. Chavez A, Scheiman J, Vora S, Pruitt BW, Tuttle M, et al. Highly efficient Cas9-mediated transcriptional programming. Nat Methods. (2015) 12:326-8. doi: 10.1038/nmeth.3312

205. Huang YH, Su J, Lei Y, Brunetti L, Gundry MC, Zhang X, et al. DNA epigenome editing using CRISPR-Cas SunTag-directed DNMT3A. Genome Biol. (2017) 18:176. doi: 10.1186/s13059-017-1306-z 
206. Tanenbaum ME, Gilbert LA, Qi LS, Weissman JS, Vale RDA. proteintagging system for signal amplification in gene expression and fluorescence imaging. Cell. (2014) 159:635-46. doi: 10.1016/j.cell.2014. 09.039

207. Zalatan JG, Lee ME, Almeida R, Gilbert LA, Whitehead EH, La Russa $\mathrm{M}$, et al. Engineering complex synthetic transcriptional programs with CRISPR RNA scaffolds. Cell. (2015) 160:339-50. doi: 10.1016/j.cell.2014. 11.052

208. Konermann S, Brigham MD, Trevino AE, Joung J, Abudayyeh OO, Barcena C, et al. Genome-scale transcriptional activation by an engineered CRISPR-Cas9 complex. Nature. (2015) 517:583-8. doi: 10.1038/nature 14136

209. Hilton IB, D’Ippolito AM, Vockley CM, Thakore PI, Crawford GE, Reddy TE, et al. Epigenome editing by a CRISPR-Cas9-based acetyltransferase activates genes from promoters and enhancers. Nat Biotechnol. (2015) 33:510-7. doi: 10.1038/nbt.3199

210. Kearns NA, Pham H, Tabak B, Genga RM, Silverstein NJ, Garber M, et al. Functional annotation of native enhancers with a Cas9-histone demethylase fusion. Nat Methods. (2015) 12:401-3. doi: 10.1038/nmeth.3325

211. Liu KI, Ramli MN, Woo CW, Wang Y, Zhao T, Zhang X, et al. A chemicalinducible CRISPR-Cas9 system for rapid control of genome editing. Nat Chem Biol. (2016) 12:980-7. doi: 10.1038/nchembio.2179

212. Zetsche B, Volz SE, Zhang F. A split-Cas9 architecture for inducible genome editing and transcription modulation. Nat Biotechnol. (2015) 33:139-42. doi: 10.1038/nbt.3149
213. Gangopadhyay SA, Cox KJ, Manna D, Lim D, Maji B, Zhou Q, et al. Precision control of CRISPR-Cas9 using small molecules and light. Biochemistry. (2019) 58:234-44. doi: 10.1021/acs.biochem.8b01202

214. Iwamoto M, Bjorklund T, Lundberg C, Kirik D, Wandless TJA. general chemical method to regulate protein stability in the mammalian central nervous system. Chem Biol. (2010) 17:981-8. doi: 10.1016/j.chembiol.2010. 07.009

215. Miyazaki Y, Imoto H, Chen LC, Wandless TJ. Destabilizing domains derived from the human estrogen receptor. J Am Chem Soc. (2012) 134:3942-5. doi: $10.1021 /$ ja209933r

216. Ravid T, Hochstrasser M. Diversity of degradation signals in the ubiquitinproteasome system. Nat Rev Mol Cell Biol. (2008) 9:679-90. doi: 10.1038/ nrm 2468

Conflict of Interest: The authors declare that the research was conducted in the absence of any commercial or financial relationships that could be construed as a potential conflict of interest.

Copyright (C) 2020 Azangou-Khyavy, Ghasemi, Khanali, Boroomand-Saboor, Jamalkhah, Soleimani and Kiani. This is an open-access article distributed under the terms of the Creative Commons Attribution License (CC BY). The use, distribution or reproduction in other forums is permitted, provided the original author(s) and the copyright owner(s) are credited and that the original publication in this journal is cited, in accordance with accepted academic practice. No use, distribution or reproduction is permitted which does not comply with these terms. 